\title{
A CONSTRUCTION OF ACTIONS ON KIRCHBERG ALGEBRAS WHICH INDUCE GIVEN ACTIONS ON THEIR K-GROUPS.
}

\author{
TAKESHI KATSURA
}

\begin{abstract}
We prove that every action of a finite group all of whose Sylow subgroups are cyclic on the $K$-theory of a Kirchberg algebra can be lifted to an action on the Kirchberg algebra. The proof uses a construction of Kirchberg algebras generalizing the one of Cuntz-Krieger algebras, and a result on modules over finite groups. As a corollary, every automorphism of the $K$-theory of a Kirchberg algebra can be lifted to an automorphism of the Kirchberg algebra with same order.
\end{abstract}

\section{INTRODUCTION}

In this paper, we consider the problem of lifting a given action on the $K$-theory of a Kirchberg algebra $\mathcal{A}$ to an action on $\mathcal{A}$. We note that in this paper a Kirchberg algebra means a simple, separable, nuclear, purely infinite $C^{*}$-algebra in the UCT class. First we state the problem more precisely.

By the theorem of Kirchberg and Phillips in [Ki, Ph], Kirchberg algebras $\mathcal{A}$ are classified by their $K$-theory $K_{*}(\mathcal{A})$ (see Subsection 1.4 for the definition). They also showed that the natural homomorphism $K_{*}: \operatorname{Aut}(\mathcal{A}) \rightarrow \operatorname{Aut}\left(K_{*}(\mathcal{A})\right)$ is surjective for every Kirchberg algebra $\mathcal{A}$. An action of a group $\Gamma$ on a Kirchberg algebra $\mathcal{A}$ induces an action of $\Gamma$ on $K_{*}(\mathcal{A})$ by composing the map $K_{*}$. The natural question asks whether we can get every action of $\Gamma$ on $K_{*}(\mathcal{A})$ in this way. This question can be restated as follows:

Lifting Problem. Let $\Gamma$ be a group and $\mathcal{A}$ be a Kirchberg algebra. Does every action of $\Gamma$ on $K_{*}(\mathcal{A})$ lift to one on $\mathcal{A}$ ?

To the author's best knowledge, no counterexample to this problem has been found so far. If one fixes a Kirchberg algebra $\mathcal{A}$ and considers the lifting problem for arbitrary groups $\Gamma$, then one leads to the following splitting problem:

Splitting Problem. Does the surjection $K_{*}: \operatorname{Aut}(\mathcal{A}) \rightarrow \operatorname{Aut}\left(K_{*}(\mathcal{A})\right)$ split?

This problem has been solved affirmatively for very few Kirchberg algebras $\mathcal{A}$. We give results (Propositions 3.15, 3.16, 3.17) on the splitting problem as corollaries of our main theorem stated below. In this paper, we consider, instead of the splitting problem, the problem to find a group $\Gamma$ for which the lifting problem has an affirmative answer for arbitrary Kirchberg algebras. The group $\Gamma=\mathbb{Z}$ satisfies this property because the map $K_{*}: \operatorname{Aut}(\mathcal{A}) \rightarrow \operatorname{Aut}\left(K_{*}(\mathcal{A})\right)$ is surjective. For a finite group $\Gamma$, as far as the author knows, the first result was due to Benson, Kumjian and Phillips who solved in $[\mathrm{BKP}]$ the lifting problem affirmatively for $\Gamma=\mathbb{Z} / 2 \mathbb{Z}$ and for unital Kirchberg algebras $\mathcal{A}$ in the Cuntz standard form. This result was extended by Spielberg who showed in [Sp] that the

2000 Mathematics Subject Classification. Primary 46L55; Secondary 46L40, 46L80.

Key words and phrases. $C^{*}$-algebra; Kirchberg algebra; group action; $K$-theory; Cuntz-Krieger algebra; group module.

The author is partially supported by JSPS Research Fellow. 
lifting problem has an affirmative answer for $\Gamma=\mathbb{Z} / p \mathbb{Z}$ where $p$ is a prime number and for an arbitrary Kirchberg algebra. See also [I] for another result on this problem using the Rohlin property. The following is the main theorem of this paper which extends the results in $[\mathrm{BKP}]$ and $[\mathrm{Sp}]$.

Theorem (Theorem 3.5). For a finite group $\Gamma$ all of whose Sylow subgroups are cyclic and an arbitrary Kirchberg algebra $\mathcal{A}$, the lifting problem has an affirmative answer.

Since a finite cyclic group $\mathbb{Z} / n \mathbb{Z}$ satisfies the assumption in the main theorem, we get the following corollary.

Corollary (Corollary 3.6). Let $\mathcal{A}$ be a Kirchberg algebra. Then every automorphism of $K_{*}(\mathcal{A})$ can be lifted to an automorphism of $\mathcal{A}$ with same order.

There are two ingredients of the proof of the main theorem. The first one is a construction of Kirchberg algebras $\mathcal{O}_{A, B}$ using generators and relations coming from two matrices $A, B \in M_{N}(\mathbb{Z})$. This construction can be considered as a generalization of the one of Cuntz-Krieger algebras introduced in [CK]. We note that a similar construction can be found in $[\mathrm{D}]$. The good points of our construction are that we can construct an action of a certain group on our $C^{*}$-algebra $\mathcal{O}_{A, B}$ by permuting generators, and we can compute the $K$-theory $K_{*}\left(\mathcal{O}_{A, B}\right)$ as well as the induced action on it using two matrices $A, B$ (Proposition 2.6). This enables us to reduce the lifting problem to a problem on modules over finite groups (Theorem 3.3). The other ingredient is the result in [Ka5] which says that all modules have an affirmative answer to this problem for a finite group all of whose Sylow subgroups are cyclic (Theorem 1.6). Combining these two results, we get the main theorem stated above.

This paper is organized as follows. Section 1 is devoted to preparation of some notation and results we need in the rest of this paper. In Section 2, we introduce the construction of a $C^{*}$-algebra $\mathcal{O}_{A, B}$ from two matrices $A, B \in M_{N}(\mathbb{Z})$ satisfying a certain condition. We also define a group $\Gamma_{A, B}$ and its action on our $C^{*}$-algebra $\mathcal{O}_{A, B}$. We state three results on $\mathcal{O}_{A, B}$ which say that we can compute the $K$-theory $K_{*}\left(\mathcal{O}_{A, B}\right)$ and the induced action of $\Gamma_{A, B}$ on it using two matrices $A, B \in M_{N}(\mathbb{Z})$ (Proposition 2.6), $\mathcal{O}_{A, B}$ is always separable, nuclear and in the UCT class (Proposition 2.9), and it is a Kirchberg algebra if $A, B$ satisfy certain conditions (Proposition 2.10). Some parts of these results can be deduced from known results as explained in Section 4. The author tries to make this paper selfcontained as much as possible, and complete and direct proofs of all the three results are provided in the latter half of this paper. In Section 3, we prove the main theorem using the construction and results in Section 2 and the result on group modules from [Ka5]. We also give several examples to which we can or cannot apply the main theorem, and show results on the splitting problem (Propositions 3.15, 3.16, 3.17). In Section 4, we examine relations of our $C^{*}$-algebras $\mathcal{O}_{A, B}$ and the Cuntz-Krieger algebras $\mathcal{O}_{A}$, the topological graph algebras $\mathcal{O}\left(E_{A, B}\right)$ and the Cuntz-Pimsner algebras $\mathcal{O}_{\mathrm{x}_{A, B}}$. We also explain which results in existing papers show which parts of the three results stated in Section 2.

In the rest of three sections, we give direct proofs of the three results on our $C^{*}$-algebras $\mathcal{O}_{A, B}$ in Section 2. In Section 5, we see that $\mathcal{O}_{A, B}$ is always nuclear by examining the so-called core $\mathcal{O}_{A, B}^{\mathbb{T}}$ of $\mathcal{O}_{A, B}$. Using this analysis, we show in Section 6 that $\mathcal{O}_{A, B}$ becomes a Kirchberg algebra when $A, B \in M_{N}(\mathbb{Z})$ satisfy certain three conditions. Finally in Section 7 , we compute the $K$-theory of $\mathcal{O}_{A, B}$ and an action of the group $\Gamma_{A, B}$ on it induced by the action $\Gamma_{A, B} \curvearrowright \mathcal{O}_{A, B}$ defined in Section 2. In the computation of the 
$K$-theories, we leave the proofs of two facts to two appendices. In Appendix A, we prove Lemma 7.6 which can be shown using [Pi, Theorem 4.4]. We try to make the proof very concrete, and to avoid $K K$-theory as much as possible. In Appendix B, we prove the last part of the proof of Proposition 7.10. Although this part is not used in the proof of the main theorem (see Remark 7.11), it is useful for examining several examples.

\section{Preliminaries}

In this section, we prepare some notation and results.

1.1. Notation. We denote by $\mathbb{C}$ the set of complex numbers, and by $\mathbb{T} \subset \mathbb{C}$ the group consisting of complex numbers with absolute values 1 . We denote by $\mathbb{Z}$ the abelian group of integers, and by $\mathbb{N}=\{0,1, \ldots\} \subset \mathbb{Z}$ the set of natural numbers. Let $\mathfrak{C}$ be the set of non-zero countable cardinalities which consists of positive integers $\{1,2, \ldots\}$ and the infinite countable cardinality $\infty$. For $N \in \mathfrak{C},\{1,2, \ldots, N\}$ is a set with cardinality $N$. Here we promise that for $N=\infty,\{1,2, \ldots, N\}$ means the set of positive integers $\{1,2, \ldots\}$ similarly as $\left\{p_{i}\right\}_{i=1}^{\infty}$ usually means $\left\{p_{1}, p_{2}, \ldots\right\}$. We extend the addition on the set of positive integers to $\mathfrak{C}$ by setting $\infty+N=N+\infty=\infty$ for $N \in \mathfrak{C}$. Then the set $\left\{1,2, \ldots, N+N^{\prime}\right\}$ has the same cardinality as $\{1,2, \ldots, N\} \amalg\left\{1,2, \ldots, N^{\prime}\right\}$ for $N, N^{\prime} \in \mathfrak{C}$.

For a set $X$, we denote by $\ell^{2}(X)$ the Hilbert space whose complete orthonormal system is given by $\left\{\delta_{x}\right\}_{x \in X}$. For a Hilbert space $H$, we denote by $K(H)$ and $B(H)$ the $C^{*}$-algebras of all compact operators and all bounded operators on $H$, respectively. For a subset $X$ of a $C^{*}$-algebra, span $X$ denotes the linear span of $X$, and $\overline{\operatorname{span}} X$ denotes its closure.

We denote by $\operatorname{Aut}(X)$ the group of automorphisms of a mathematical object $X$, such as a set, an abelian group or a $C^{*}$-algebra. An action $\Gamma \curvearrowright X$ of a group $\Gamma$ on a mathematical object $X$ is a homomorphism from $\Gamma$ to $\operatorname{Aut}(X)$. For an action $\Gamma \curvearrowright X$, the automorphism of $X$ defined by an element $\gamma \in \Gamma$ is often denoted by the same symbol $\gamma$. An abelian group $G$ with an action of $\Gamma$ is called a $\Gamma$-module. An isomorphism as $\Gamma$-modules means an isomorphism as abelian groups which is $\Gamma$-equivariant.

1.2. Permutation presentations of modules. Let us take $N \in \mathfrak{C}$. Let $\mathbb{Z}^{N}$ be the free abelian group whose basis is given by $\left\{e_{i}\right\}_{i=1}^{N}$. If an action $\Gamma \curvearrowright\{1,2, \ldots, N\}$ is given, we can define an action $\Gamma \curvearrowright \mathbb{Z}^{N}$ by $\gamma\left(e_{i}\right)=e_{\gamma(i)}$. Thus we get a $\Gamma$-module $\mathbb{Z}^{N}$. We call such a module a countable permutation $\Gamma$-module.

Definition 1.1. A countable permutation presentation of a $\Gamma$-module $G$ is a $\Gamma$-equivariant exact sequence

$$
0 \longrightarrow F \longrightarrow F \longrightarrow 0
$$

where $F$ is a countable permutation $\Gamma$-module.

We are going to see that a countable permutation presentation is presented by a $N \times N$ matrix with integer entries for some $N \in \mathfrak{C}$.

For a positive integer $N, M_{N}(\mathbb{Z})$ denotes the algebra of all $N \times N$ matrices with integer entries. For $N=\infty, M_{\infty}(\mathbb{Z})$ denotes the algebra of all infinite matrices $D=\left(D_{i, j}\right)_{i, j=1}^{\infty}$ with integer entries satisfying that the set

$$
\left\{j \in\{1,2, \ldots\} \mid D_{i, j} \neq 0\right\}
$$

is finite for all $i$. 
Let $N \in \mathfrak{C}$. A homomorphism $\varphi: \mathbb{Z}^{N} \rightarrow \mathbb{Z}^{N}$ is represented by an element $D \in M_{N}(\mathbb{Z})$ so that $\varphi\left(e_{i}\right)=\sum_{j=1}^{N} D_{i, j} e_{j}$, and by this correspondence we can and will identify the set $M_{N}(\mathbb{Z})$ and the set of all endomorphisms of $\mathbb{Z}^{N}$. Note that we consider $\mathbb{Z}^{N}$ as the set of row vectors via

$$
\left(n_{1}, n_{2}, \ldots, n_{N}\right) \mapsto \sum_{i=1}^{N} n_{i} e_{i} \in \mathbb{Z}^{N},
$$

and think that $M_{N}(\mathbb{Z})$ acts $\mathbb{Z}^{N}$ from right. We denote by $\mathbb{N}^{N}$ the subset of $\mathbb{Z}^{N}$ consisting of elements in the form $f=\sum_{i=1}^{N} n_{i} e_{i} \in \mathbb{Z}^{N}$ with $n_{i} \in \mathbb{N}$ for all $i$, and by $M_{N}(\mathbb{N})$ the subset of $M_{N}(\mathbb{Z})$ consisting of the matrices whose entries are in $\mathbb{N}$. The identity matrix of $M_{N}(\mathbb{Z})$ is denote by $I \in M_{N}(\mathbb{Z})$.

Let us take an action $\Gamma \curvearrowright\{1,2, \ldots, N\}$. This action makes $\mathbb{Z}^{N}$ a countable permutation $\Gamma$-module. We set

$$
M_{N}(\mathbb{Z})^{\Gamma}:=\left\{D \in M_{N}(\mathbb{Z}) \mid D_{i, j}=D_{\gamma(i), \gamma(j)} \text { for all } i, j \text { and } \gamma \in \Gamma\right\} .
$$

It is easy to see that a matrix $D$ is in $M_{N}(\mathbb{Z})^{\Gamma}$ if and only if the homomorphism $\mathbb{Z}^{N} \rightarrow \mathbb{Z}^{N}$ determined by $D$ is $\Gamma$-equivariant. For $D \in M_{N}(\mathbb{Z})^{\Gamma}$, the action $\Gamma \curvearrowright \mathbb{Z}^{N}$ induces actions of $\Gamma$ on the abelian groups ker $D$ and coker $D$. Thus ker $D$ and coker $D$ become $\Gamma$-modules. We will denote by $[\cdot]$ the natural surjection $\mathbb{Z}^{N} \rightarrow$ coker $D$. The following observation is easy to see.

Lemma 1.2. Giving a countable permutation presentation of a $\Gamma$-module $G$ is same as giving $N \in \mathfrak{C}$, an action $\Gamma \curvearrowright\{1,2, \ldots, N\}$, a matrix $D \in M_{N}(\mathbb{Z})^{\Gamma}$ with $\operatorname{ker} D=0$ and an isomorphism coker $D \cong G$ as $\Gamma$-modules.

Example 1.3. Let $\Gamma_{1} \cong \mathbb{Z} / 2 \mathbb{Z}$ be a cyclic group generated by $\sigma$ with $\sigma^{2}=1$. Let $G=\mathbb{Z} / 3 \mathbb{Z}$ be a $\Gamma_{1}$-module where an action of $\Gamma_{1}$ on $G$ is defined by $\sigma(g)=-g$. Then $G$ has a countable permutation presentation which is represented by $N=2$, an action of $\Gamma_{1}$ on $\{1,2\}$ defined by $\sigma(1)=2$ and $\sigma(2)=1$, a $2 \times 2$-matrix

$$
D=\left(\begin{array}{cc}
2 & -1 \\
-1 & 2
\end{array}\right) \in M_{2}(\mathbb{Z})^{\Gamma_{1}}
$$

with $\operatorname{ker} D=0$, and an isomorphism coker $D \cong G$ defined by $\left[e_{i}\right] \mapsto i$ for $i=1,2$.

Example 1.4. Let $\Gamma_{2} \cong(\mathbb{Z} / 2 \mathbb{Z}) \times(\mathbb{Z} / 2 \mathbb{Z})$ be a group generated by two elements $\sigma, \tau \in \Gamma_{2}$ with relations $\sigma^{2}=\tau^{2}=1$ and $\sigma \tau=\tau \sigma$. Let $G=(\mathbb{Z} / 3 \mathbb{Z})^{3}$ be a $\Gamma_{2}$-module where an action of $\Gamma_{2}$ on $G$ is defined by

$$
\sigma\left(\left(a_{1}, a_{2}, a_{3}\right)\right)=\left(a_{2}, a_{1},-a_{1}-a_{2}-a_{3}\right), \quad \tau\left(\left(a_{1}, a_{2}, a_{3}\right)\right)=\left(a_{3},-a_{1}-a_{2}-a_{3}, a_{1}\right),
$$

for $a_{1}, a_{2}, a_{3} \in \mathbb{Z} / 3 \mathbb{Z}$. This $\Gamma_{2}$-module $G$ has a countable permutation presentation which is represented by $N=4$, an action of $\Gamma_{2}$ on $\{1,2,3,4\}$ defined by

$$
\begin{array}{cccc}
\sigma: 1 \mapsto 2, & 2 \mapsto 1, & 3 \mapsto 4, & 4 \mapsto 3, \\
\tau: 1 \mapsto 3, & 2 \mapsto 4, & 3 \mapsto 1, & 4 \mapsto 2,
\end{array}
$$

a matrix

$$
D=\left(\begin{array}{cccc}
2 & -1 & -1 & -1 \\
-1 & 2 & -1 & -1 \\
-1 & -1 & 2 & -1 \\
-1 & -1 & -1 & 2
\end{array}\right) \in M_{4}(\mathbb{Z})^{\Gamma_{2}}
$$


with $\operatorname{ker} D=0$ and an isomorphism coker $D \cong G$ defined by

$$
\left[\sum_{i=1}^{4} n_{i} e_{i}\right] \mapsto\left(n_{1}+n_{2}+n_{3}, n_{1}+n_{2}+n_{4}, n_{1}+n_{3}+n_{4}\right) .
$$

Example 1.5. The same formula as in Example 1.4 defines an action of $\Gamma_{2}$ on $G^{\prime}=$ $(\mathbb{Z} / 4 \mathbb{Z})^{3}$. We can show that this $\Gamma_{2}$-module $G^{\prime}$ has no countable permutation presentations (see [Ka5, Example 2.6]).

In $[\mathrm{Ka} 5]$, we prove the following.

Theorem 1.6 ([Ka5, Proposition 1.3 and Theorem 1.4]). Let $\Gamma$ be a finite group all of whose Sylow subgroups are cyclic. Then every countable $\Gamma$-module has a countable permutation presentation.

Note that if a finite group $\Gamma$ has a non-cyclic Sylow subgroup, then the countable $\Gamma$ module called the augmentation ideal of $\Gamma$ has no countable permutation presentations (see [Ka5, Proposition 2.8]).

1.3. $\boldsymbol{K}$-groups and partial unitaries. Let $\mathcal{A}$ be a $C^{*}$-algebra. For definitions and results of $K$-groups $K_{0}(\mathcal{A})$ and $K_{1}(\mathcal{A})$, we consult the book [Bl]. We denote by $[p]$ the element in $K_{0}(\mathcal{A})$ defined by a projection $p \in \mathcal{A}$. For a projection $p \in \mathcal{A}$, we say that $u$ is a partial unitary with $u^{0}=p$ if $u^{*} u=u u^{*}=p$. We denote by $\widetilde{\mathcal{A}}$ the minimal unital $C^{*}$-algebra containing $\mathcal{A}$. For a partial unitary $u \in \mathcal{A}$ with $u^{0}=p$, we denote by $\widetilde{u} \in \widetilde{\mathcal{A}}$ the unitary $u+(1-p)$. The element in $K_{1}(\mathcal{A})$ defined by this unitary $\widetilde{u}$ is denoted by $[u]$. For a partial unitary $u \in \mathcal{A}$ with $u^{0}=p$, we define $u^{n} \in \mathcal{A}$ for $n \in \mathbb{Z}$ by

$$
u^{n}= \begin{cases}u^{n} & \text { for } n>0 \\ p & \text { for } n=0 \\ \left(u^{*}\right)^{-n} & \text { for } n<0\end{cases}
$$

Then $u^{n} \in \mathcal{A}$ is also a partial unitary with $\left(u^{n}\right)^{0}=p$ and satisfies $\widetilde{u^{n}}=\widetilde{u}^{n}$ for $n \in \mathbb{Z}$. For a finite family $\left\{u_{i}\right\}_{i}$ of mutually orthogonal partial unitaries in $\mathcal{A}$, the element $u=\sum_{i} u_{i} \in$ $\mathcal{A}$ is a partial unitary. The family $\left\{\widetilde{u}_{i}\right\}_{i}$ of unitaries in $\widetilde{\mathcal{A}}$ is mutually commutative, and we have $\widetilde{u}=\prod_{i} \widetilde{u}_{i}$. Hence we get $[u]=\sum_{i}\left[u_{i}\right]$ in $K_{1}(\mathcal{A})$.

\subsection{Kirchberg algebras.}

Definition 1.7. A Kirchberg algebra is a simple, separable, nuclear, purely infinite $C^{*}$ algebra in the UCT class.

For a detailed definition and results of Kirchberg algebras, we consult the book [Rø]. We remark that in $[R \varnothing$, Definition 4.3.1] or some literatures, Kirchberg algebras are not assume to be in the UCT class.

For a Kirchberg algebra $\mathcal{A}$, we define $K_{*}(\mathcal{A})$ by

$$
K_{*}(\mathcal{A})= \begin{cases}\left(K_{0}(\mathcal{A}), K_{1}(\mathcal{A})\right) & \text { if } \mathcal{A} \text { is stable } \\ \left.\left(K_{0}(\mathcal{A}), 1_{\mathcal{A}}\right], K_{1}(\mathcal{A})\right) & \text { if } \mathcal{A} \text { has a unit } 1_{\mathcal{A}}\end{cases}
$$

Recall that a Kirchberg algebra is stable if and only if it is non-unital ([Z]).

Let $G_{0}, G_{1}, G_{0}^{\prime}$ and $G_{1}^{\prime}$ be countable abelian groups. An isomorphism of the two pairs $\left(G_{0}, G_{1}\right)$ and $\left(G_{0}^{\prime}, G_{1}^{\prime}\right)$ is defined to be a pair $\varphi_{*}=\left(\varphi_{0}, \varphi_{1}\right)$ of isomorphisms $\varphi_{i}: G_{i} \rightarrow G_{i}^{\prime}$ 
for $i=0,1$. If such an isomorphism exists, we write $\left(G_{0}, G_{1}\right) \cong\left(G_{0}^{\prime}, G_{1}^{\prime}\right)$. Let us take $g \in G_{0}$ and $g^{\prime} \in G_{0}^{\prime}$. An isomorphism of $\left(G_{0}, g, G_{1}\right)$ and $\left(G_{0}^{\prime}, g^{\prime}, G_{1}^{\prime}\right)$ is defined to be a pair $\varphi_{*}=\left(\varphi_{0}, \varphi_{1}\right)$ of isomorphisms $\varphi_{i}: G_{i} \rightarrow G_{i}^{\prime}$ for $i=0,1$ with $\varphi_{0}(g)=g^{\prime}$. If such an isomorphism exists, we write $\left(G_{0}, g, G_{1}\right) \cong\left(G_{0}^{\prime}, g^{\prime}, G_{1}^{\prime}\right)$. With these preparations, we can state the celebrated classification theorem due to Elliott, Kirchberg, Phillips and Rørdam as follows (see [Rø, Proposition 4.3.3, Theorem 8.4.1]).

Theorem 1.8. Let $\left(G_{0}, G_{1}\right)$ be a pair of countable abelian groups. Then there exists a unique stable Kirchberg algebra $\mathcal{A}$ with $K_{*}(\mathcal{A}) \cong\left(G_{0}, G_{1}\right)$, and for each $g \in G_{0}$ there exists a unique unital Kirchberg algebra $\mathcal{A}$ with $K_{*}(\mathcal{A}) \cong\left(G_{0}, g, G_{1}\right)$.

On the line of the proof of the theorem above, the natural homomorphism $\operatorname{Aut}(\mathcal{A}) \rightarrow$ $\operatorname{Aut}\left(K_{*}(\mathcal{A})\right)$ is shown to be surjective for a Kirchberg algebra $\mathcal{A}$. By composing this surjection, an action of a group $\Gamma$ on a Kirchberg algebra $\mathcal{A}$ induces an action of $\Gamma$ on the $K$-theory $K_{*}(\mathcal{A})$ of $\mathcal{A}$. In particular, the two groups $K_{0}(\mathcal{A})$ and $K_{1}(\mathcal{A})$ become $\Gamma$-modules.

\section{The $C^{*}$-Algebra $\mathcal{O}_{A, B}$}

In this section, we construct a $C^{*}$-algebra $\mathcal{O}_{A, B}$, a group $\Gamma_{A, B}$ and an action $\Gamma_{A, B} \curvearrowright$ $\mathcal{O}_{A, B}$ from two matrices $A \in M_{N}(\mathbb{N})$ and $B \in M_{N}(\mathbb{Z})$. We also state three results on $\mathcal{O}_{A, B}$ which will be proven in the latter half of this paper.

Definition 2.1. Let $N \in \mathfrak{C}$. For $A \in M_{N}(\mathbb{N})$, we define a set $\Omega_{A}$ by

$$
\Omega_{A}:=\left\{(i, j) \in\{1,2, \ldots, N\} \times\{1,2, \ldots, N\} \mid A_{i, j} \geq 1\right\} .
$$

For each $i \in\{1,2, \ldots, N\}$, we define a set $\Omega_{A}(i) \subset\{1,2, \ldots, N\}$ by

$$
\Omega_{A}(i):=\left\{j \in\{1,2, \ldots, N\} \mid(i, j) \in \Omega_{A}\right\} .
$$

Note that by definition $\Omega_{A}(i)$ is finite for all $i$.

Definition 2.2. Let us take $N \in \mathfrak{C}, A \in M_{N}(\mathbb{N})$ and $B \in M_{N}(\mathbb{Z})$. We define a $C^{*}$-algebra $\mathcal{O}_{A, B}$ to be the universal $C^{*}$-algebra generated by mutually orthogonal projections $\left\{p_{i}\right\}_{i=1}^{N}$, partial unitaries $\left\{u_{i}\right\}_{i=1}^{N}$ with $u_{i}^{0}=p_{i}$, and partial isometries $\left\{s(n)_{i, j}\right\}_{(i, j) \in \Omega_{A}, n \in \mathbb{Z}}$ satisfying the relations

(i) $s(n)_{i, j} u_{j}=s\left(n+A_{i, j}\right)_{i, j}$ and $u_{i} s(n)_{i, j}=s\left(n+B_{i, j}\right)_{i, j}$ for all $(i, j) \in \Omega_{A}$ and $n \in \mathbb{Z}$,

(ii) $s(n)_{i, j}^{*} s(n)_{i, j}=p_{j}$ for all $(i, j) \in \Omega_{A}$ and $n \in \mathbb{Z}$,

(iii) $p_{i}=\sum_{j \in \Omega_{A}(i)} \sum_{n=1}^{A_{i, j}} s(n)_{i, j} s(n)_{i, j}^{*}$ for all $i$.

If there exists $i$ with $\Omega_{A}(i)=\emptyset$, then the condition (iii) says that $p_{i}=0$. Hence $\mathcal{O}_{A, B}$ is isomorphic to $\mathcal{O}_{A^{\prime}, B^{\prime}}$ where $A^{\prime}, B^{\prime} \in M_{N-1}(\mathbb{Z})$ are obtained by eliminating the $i$-th rows and the $i$-th columns from $A, B$. By repeating this argument as many as possible, either we get $\mathcal{O}_{A, B}=0$, or we can find $A^{\prime} \in M_{N^{\prime}}(\mathbb{N})$ and $B^{\prime} \in M_{N^{\prime}}(\mathbb{Z})$ which are isomorphic to corners of $A$ and $B$ such that $\Omega_{A^{\prime}}(i) \neq \emptyset$ for all $i$ and $\mathcal{O}_{A, B} \cong \mathcal{O}_{A^{\prime}, B^{\prime}}$ naturally. Hence without loss of generality, we may assume that $\Omega_{A}(i) \neq \emptyset$ for all $i$. In Definition 2.2, we use $B_{i, j} \in \mathbb{Z}$ only for $(i, j) \in \Omega_{A}$. Hence without loss of generality, we may assume that $(i, j) \notin \Omega_{A}$ implies $B_{i, j}=0$. We summarize these assumptions into the following condition on $A, B \in M_{N}(\mathbb{Z})$ for further reference;

(0) $A \in M_{N}(\mathbb{N}), \Omega_{A}(i) \neq \emptyset$ for all $i$, and $B_{i, j}=0$ for $(i, j) \notin \Omega_{A}$. 
Remark 2.3. As we did when defining graph algebras (see [Ra]), we can change "for all $i$ " in the condition (iii) to "for all $i$ with $\Omega_{A}(i) \neq \emptyset$ " in order to get a meaningful $C^{*}$-algebra $\mathcal{O}_{A, B}$ for $A \in M_{N}(\mathbb{N})$ and $B \in M_{N}(\mathbb{Z})$ with $\Omega_{A}(i)=\emptyset$ for some $i$. One can also drop the assumption that $\Omega_{A}(i)$ is finite by changing the condition (iii) suitably (cf. [Ka4, Proposition B.2]). For our purpose in this paper we do not need such a generality, and hence we only consider $A, B \in M_{N}(\mathbb{Z})$ satisfying the condition (0).

Definition 2.4. Let us take $N \in \mathfrak{C}$, and $A, B \in M_{N}(\mathbb{Z})$. We define a group $\Gamma_{A, B}$ by

$$
\Gamma_{A, B}:=\left\{\gamma \in \operatorname{Aut}(\{1,2, \ldots, N\}) \mid A_{i, j}=A_{\gamma(i), \gamma(j)} \text { and } B_{i, j}=B_{\gamma(i), \gamma(j)} \text { for all } i, j\right\} \text {. }
$$

By definition, $\Gamma_{A, B}$ acts on $\{1,2, \ldots, N\}$ and we have $A, B \in M_{N}(\mathbb{Z})^{\Gamma_{A, B}}$. If we have $A, B \in M_{N}(\mathbb{Z})^{\Gamma}$ for an action of some group $\Gamma$ on $\{1,2, \ldots, N\}$, then there exists a unique homomorphism $\Gamma \rightarrow \Gamma_{A, B}$ such that the action of $\Gamma$ is the composition of this homomorphism and the action of $\Gamma_{A, B}$.

Definition 2.5. Let $A, B \in M_{N}(\mathbb{Z})$ satisfy the condition (0) above. We define an action $\Gamma_{A, B} \curvearrowright \mathcal{O}_{A, B}$ by

$$
\gamma\left(p_{i}\right)=p_{\gamma(i)}, \quad \gamma\left(u_{i}\right)=u_{\gamma(i)} \quad \text { and } \quad \gamma\left(s(n)_{i, j}\right)=s(n)_{\gamma(i), \gamma(j)}
$$

for the generators $\left\{p_{i}, u_{i}, s(n)_{i, j}\right\}$ of the $C^{*}$-algebra $\mathcal{O}_{A, B}$ and $\gamma \in \Gamma_{A, B}$.

It is routine to check that the definition above is well-defined. This action induces actions of $\Gamma_{A, B}$ on $K_{0}\left(\mathcal{O}_{A, B}\right)$ and $K_{1}\left(\mathcal{O}_{A, B}\right)$. On the other hand, since $I-A, I-B \in$ $M_{N}(\mathbb{Z})^{\Gamma_{A, B}}$, we get actions of $\Gamma_{A, B}$ on $\operatorname{ker}(I-A), \operatorname{coker}(I-A), \operatorname{ker}(I-B)$ and coker $(I-B)$. We obtain the following whose proof can be found in Section 7.

Proposition 2.6. There exist $\Gamma_{A, B}$-equivariant isomorphisms

$$
\begin{aligned}
& K_{0}\left(\mathcal{O}_{A, B}\right) \cong \operatorname{coker}(I-A) \oplus \operatorname{ker}(I-B), \\
& K_{1}\left(\mathcal{O}_{A, B}\right) \cong \operatorname{coker}(I-B) \oplus \operatorname{ker}(I-A)
\end{aligned}
$$

under which $\left[p_{i}\right] \in K_{0}\left(\mathcal{O}_{A, B}\right)$ and $\left[u_{i}\right] \in K_{1}\left(\mathcal{O}_{A, B}\right)$ correspond to $\left[e_{i}\right] \in \operatorname{coker}(I-A)$ and $\left[e_{i}\right] \in \operatorname{coker}(I-B)$ respectively for every $i$.

Proof. This follows from Proposition 7.10 and its proof.

Corollary 2.7. Let $A, B \in M_{N}(\mathbb{Z})$ satisfy the condition (0) above. Let $\Gamma \curvearrowright\{1,2, \ldots, N\}$ be an action with $A, B \in M_{N}(\mathbb{Z})^{\Gamma}$ which naturally induces the actions of $\Gamma$ on $\operatorname{ker}(I-A)$, $\operatorname{coker}(I-A), \operatorname{ker}(I-B)$ and $\operatorname{coker}(I-B)$. Then we have the natural action $\Gamma \curvearrowright \mathcal{O}_{A, B}$ such that the isomorphisms in Proposition 2.6 are $\Gamma$-equivariant.

Remark 2.8. For $A, B \in M_{N}(\mathbb{Z})$ satisfying the condition (0), the $C^{*}$-algebra $\mathcal{O}_{A, B}$ is unital if and only if $N<\infty$, and in this case the unit is $\sum_{i=1}^{N} p_{i} \in \mathcal{O}_{A, B}$ (see Subsection 4.1). Thus for $N<\infty,\left[1_{\mathcal{O}_{A, B}}\right] \in K_{0}\left(\mathcal{O}_{A, B}\right)$ corresponds to $\left[\sum_{i=1}^{N} e_{i}\right] \in \operatorname{coker}(I-A)$ under the isomorphism in Proposition 2.6.

We also get the following two propositions which will be proven in Sections 5, 6 and 7 .

Proposition 2.9. For $A, B \in M_{N}(\mathbb{Z})$ satisfying the condition (0), the $C^{*}$-algebra $\mathcal{O}_{A, B}$ is separable, nuclear and in the UCT class.

Proof. The $C^{*}$-algebra $\mathcal{O}_{A, B}$ is separable because its generator is countable. It is nuclear by Proposition 5.6, and in the UCT class by Proposition 7.8. 
Proposition 2.10. If $A, B \in M_{N}(\mathbb{Z})$ satisfy the condition (0) and the conditions

(1) $A \in M_{N}(\mathbb{N})$ is irreducible, i.e. for every $i, j \in\{1,2, \ldots, N\}$ there exists a positive integer $n$ with $\left(A^{n}\right)_{i, j} \geq 1$,

(2) $A_{i, i} \geq 2$ and $B_{i, i}=1$ for every $i \in\{1,2, \ldots, N\}$,

then the $C^{*}$-algebra $\mathcal{O}_{A, B}$ is simple and purely infinite, and hence a Kirchberg algebra by Proposition 2.9.

Proof. See Section 6.

\section{The PROOF OF THE MAIN THEOREM}

In this section, we prove the main theorem (Theorem 3.5) using the results in the previous section and Theorem 1.6. The following lemma is an equivariant version of [Ka4, Lemma 6.4].

Lemma 3.1. Let $\Gamma$ be a finite group. For $N^{\prime} \in \mathfrak{C}$, an action $\Gamma \curvearrowright\left\{1,2, \ldots, N^{\prime}\right\}$ and $A^{\prime}, B^{\prime} \in M_{N^{\prime}}(\mathbb{Z})^{\Gamma}$, there exist $N \in \mathfrak{C}$, an action $\Gamma \curvearrowright\{1,2, \ldots, N\}$ and $A, B \in M_{N}(\mathbb{Z})^{\Gamma}$ satisfying the conditions (0), (1), (2) in Section 2 such that there exist $\Gamma$-equivariant isomorphisms

$$
\begin{aligned}
& \operatorname{ker}(I-A) \cong \operatorname{ker} A^{\prime}, \quad \operatorname{coker}(I-A) \cong \operatorname{coker} A^{\prime}, \\
& \operatorname{ker}(I-B) \cong \operatorname{ker} B^{\prime}, \quad \quad \operatorname{coker}(I-B) \cong \operatorname{coker} B^{\prime} .
\end{aligned}
$$

Proof. In this proof, we denote by $I^{\prime} \in M_{N^{\prime}}(\mathbb{N})$ the identity matrix of $M_{N^{\prime}}(\mathbb{N})$. We define $\left|A^{\prime}\right|,\left|B^{\prime}\right| \in M_{N^{\prime}}(\mathbb{N})$ by $\left|A^{\prime}\right|_{i, j}=\left|A_{i, j}^{\prime}\right|$ and $\left|B^{\prime}\right|_{i, j}=\left|B_{i, j}^{\prime}\right|$ for $i, j \in\left\{1,2, \ldots, N^{\prime}\right\}$. We define $X \in M_{N^{\prime}}(\mathbb{N})$ by

$$
X_{i, j}= \begin{cases}1 & \text { if }|i-j|=1, \\ 0 & \text { if }|i-j| \neq 1,\end{cases}
$$

and $X^{\gamma} \in M_{N^{\prime}}(\mathbb{N})$ by $\left(X^{\gamma}\right)_{i, j}=X_{\gamma(i), \gamma(j)}$ for $\gamma \in \Gamma$. We set $Y \in M_{N^{\prime}}(\mathbb{N})$ by

$$
Y=\left|A^{\prime}\right|+\left|B^{\prime}\right|+I^{\prime}+\sum_{\gamma \in \Gamma} X^{\gamma} .
$$

Then we have $Y \in M_{N^{\prime}}(\mathbb{Z})^{\Gamma}$. We define $A, B \in M_{2}\left(M_{N^{\prime}}(\mathbb{Z})\right)$ by

$$
A=\left(\begin{array}{cc}
2 I^{\prime} & A^{\prime}+Y \\
I^{\prime} & I^{\prime}+Y
\end{array}\right), \quad B=\left(\begin{array}{cc}
I^{\prime} & B^{\prime} \\
I^{\prime} & I^{\prime}
\end{array}\right) .
$$

We set $N=N^{\prime}+N^{\prime} \in \mathfrak{C}$. Choose a bijection

$$
\left\{1,2, \ldots, N^{\prime}\right\} \amalg\left\{1,2, \ldots, N^{\prime}\right\} \cong\{1,2, \ldots, N\}
$$

and fix it. Using this bijection, we identify $\mathbb{Z}^{N^{\prime}} \oplus \mathbb{Z}^{N^{\prime}} \cong \mathbb{Z}^{N}$ and $M_{2}\left(M_{N^{\prime}}(\mathbb{Z})\right) \cong M_{N}(\mathbb{Z})$, and regard $A, B \in M_{N}(\mathbb{Z})$. Through this bijection, the action $\Gamma \curvearrowright\left\{1,2, \ldots, N^{\prime}\right\}$ induces an action $\Gamma \curvearrowright\{1,2, \ldots, N\}$. We see that $A, B \in M_{N}(\mathbb{Z})^{\Gamma}$ because all the entries of $A, B \in M_{2}\left(M_{N^{\prime}}(\mathbb{Z})\right)$ are in $M_{N^{\prime}}(\mathbb{Z})^{\Gamma}$. By noticing $A^{\prime}+\left|A^{\prime}\right| \in M_{N^{\prime}}(\mathbb{N})$, one can easily check that $A, B \in M_{N}(\mathbb{Z})$ satisfy the conditions (0) and (2). Since $X^{1}=X \in M_{N^{\prime}}(\mathbb{N})$ is irreducible, so is

$$
\left(\begin{array}{ll}
I^{\prime} & I^{\prime} \\
I^{\prime} & X^{1}
\end{array}\right) \in M_{N}(\mathbb{N})
$$

Hence $A \in M_{N}(\mathbb{N})$ is irreducible. Thus $A, B \in M_{N}(\mathbb{Z})$ also satisfy the condition (1). 
Note that the identity matrix $I \in M_{N}(\mathbb{Z})$ corresponds to

$$
\left(\begin{array}{cc}
I^{\prime} & 0 \\
0 & I^{\prime}
\end{array}\right) \in M_{2}\left(M_{N^{\prime}}(\mathbb{Z})\right)
$$

via the identification $M_{N}(\mathbb{Z}) \cong M_{2}\left(M_{N^{\prime}}(\mathbb{Z})\right)$. We have the equality

$$
\begin{aligned}
I-A & =\left(\begin{array}{cc}
-I^{\prime} & -A^{\prime}-Y \\
-I^{\prime} & -Y
\end{array}\right) \\
& =\left(\begin{array}{ll}
I^{\prime} & I^{\prime} \\
0 & I^{\prime}
\end{array}\right)\left(\begin{array}{cc}
A^{\prime} & 0 \\
0 & -I^{\prime}
\end{array}\right)\left(\begin{array}{cc}
0 & -I^{\prime} \\
I^{\prime} & Y
\end{array}\right) .
\end{aligned}
$$

Since the left and the right matrices in the multiplication above define $\Gamma$-equivariant isomorphisms from $\mathbb{Z}^{N}$ to $\mathbb{Z}^{N}$, there exist $\Gamma$-equivariant isomorphisms

$$
\begin{aligned}
& \operatorname{ker}(I-A) \cong \operatorname{ker}\left(\begin{array}{cc}
A^{\prime} & 0 \\
0 & -I^{\prime}
\end{array}\right) \cong \operatorname{ker} A^{\prime}, \\
& \operatorname{coker}(I-A) \cong \operatorname{coker}\left(\begin{array}{cc}
A^{\prime} & 0 \\
0 & -I^{\prime}
\end{array}\right) \cong \operatorname{coker} A^{\prime} .
\end{aligned}
$$

Similarly, we have $\Gamma$-equivariant isomorphisms

$$
\operatorname{ker}(I-B) \cong \operatorname{ker} B^{\prime}, \quad \operatorname{coker}(I-B) \cong \operatorname{coker} B^{\prime} .
$$

We are done.

Proposition 3.2. Let $\Gamma$ be a finite group, and $G_{0}, G_{1}$ be $\Gamma$-modules which have countable permutation presentations. Then there exist $N \in \mathfrak{C}$, an action $\Gamma \curvearrowright\{1,2, \ldots, N\}$ and $A, B \in M_{N}(\mathbb{Z})^{\Gamma}$ satisfying the conditions (0), (1), (2) in Section 2 such that $\operatorname{ker}(I-A)=$ $\operatorname{ker}(I-B)=0, \operatorname{coker}(I-A) \cong G_{0}$ and $\operatorname{coker}(I-B) \cong G_{1}$ as $\Gamma$-modules.

Moreover, for each $g \in G_{0}$ fixed by the action of $\Gamma$, we can find $f \in \mathbb{N}^{N} \subset \mathbb{Z}^{N}$ fixed by the action of $\Gamma$ such that $[f] \in \operatorname{coker}(I-A)$ corresponds to $g \in G_{0}$ under the isomorphism above.

Proof. For $i=0,1$, Lemma 1.2 gives $N_{i} \in \mathfrak{C}$, an action $\Gamma \curvearrowright\left\{1,2, \ldots, N_{i}\right\}$, a matrix $D_{i} \in M_{N_{i}}(\mathbb{Z})^{\Gamma}$ with $\operatorname{ker} D_{i}=0$ and an isomorphism coker $D_{i} \cong G_{i}$ as $\Gamma$-modules. Let $N^{\prime}=N_{0}+N_{1}$ and choose a bijection

$$
\left\{1,2, \ldots, N_{0}\right\} \amalg\left\{1,2, \ldots, N_{1}\right\} \cong\left\{1,2, \ldots, N^{\prime}\right\} .
$$

Through this bijection, two actions of $\Gamma$ on $\left\{1,2, \ldots, N_{i}\right\}$ for $i=0,1$ define an action of $\Gamma$ on $\left\{1,2, \ldots, N^{\prime}\right\}$. Let $A^{\prime}, B^{\prime} \in M_{N^{\prime}}(\mathbb{Z})^{\Gamma}$ be the images of $\left(D_{0}, I\right),\left(I, D_{1}\right) \in M_{N_{0}}(\mathbb{Z})^{\Gamma} \oplus$ $M_{N_{1}}(\mathbb{Z})^{\Gamma}$ under the natural inclusion $M_{N_{0}}(\mathbb{Z})^{\Gamma} \oplus M_{N_{1}}(\mathbb{Z})^{\Gamma} \rightarrow M_{N^{\prime}}(\mathbb{Z})^{\Gamma}$ defined from the bijection. Then we have $\operatorname{ker} A^{\prime}=\operatorname{ker} B^{\prime}=0$, coker $A^{\prime} \cong G_{0}$ and coker $B^{\prime} \cong G_{1}$ as $\Gamma$ modules. By Lemma 3.1, we get $N \in \mathfrak{C}$, an action $\Gamma \curvearrowright\{1,2, \ldots, N\}$ and $A, B \in M_{N}(\mathbb{Z})^{\Gamma}$ satisfying the conditions (0), (1), (2) in Section 2 such that $\operatorname{ker}(I-A)=\operatorname{ker}(I-B)=0$, $\operatorname{coker}(I-A) \cong G_{0}$ and $\operatorname{coker}(I-B) \cong G_{1}$ as $\Gamma$-modules.

The isomorphism $\operatorname{coker}(I-A) \cong G_{0}$ gives us a surjection $\pi: \mathbb{Z}^{N} \rightarrow G_{0}$ such that $[f] \in \operatorname{coker}(I-A)$ corresponds to $\pi(f) \in G_{0}$ for each $f \in \mathbb{Z}^{N}$. The kernel of the surjection $\pi$ is isomorphic to $\mathbb{Z}^{N}$ because $\operatorname{ker}(I-A)=0$ :

$$
0 \longrightarrow \mathbb{Z}^{N} \stackrel{I-A}{\longrightarrow} \mathbb{Z}^{N} \stackrel{\pi}{\longrightarrow} G_{0} \longrightarrow 0
$$

Take an element $g \in G_{0}$ fixed by the action of $\Gamma$. We can show that there exists $f \in \mathbb{Z}^{N}$ which is fixed by the action of $\Gamma$ and satisfies $\pi(f)=g$ because the obstruction of such a 
lifting is encoded in the group cohomology $H^{1}(\Gamma, \operatorname{ker} \pi) \cong H^{1}\left(\Gamma, \mathbb{Z}^{N}\right)$ which can be shown to vanish easily (see [Ka5, Remark 2.3 and Lemma $3.4(1)]$ ). Let us write $f=\sum_{i=1}^{N} n_{i} e_{i}$ for $n_{i} \in \mathbb{Z}$. If $n_{i} \geq 0$ for all $i$, this $f \in \mathbb{N}^{N} \subset \mathbb{Z}^{N}$ satisfies the desired condition. Suppose that there exists $i_{0}$ with $n_{i_{0}}<0$. Let $f_{i_{0}} \in \mathbb{Z}^{N}$ be the image of $e_{i_{0}} \in \mathbb{Z}^{N}$ under the map $A-I$. Then we have $f_{i_{0}}-e_{i_{0}} \in \mathbb{N}^{N}$ because $A \in M_{N}(\mathbb{N})$ and $A_{i_{0}, i_{0}} \geq 2$. Set $f^{\prime}:=f-n_{i_{0}} \sum_{\gamma \in \Gamma} \gamma\left(f_{i_{0}}\right)$. Then $f^{\prime}$ is fixed by the action of $\Gamma$ and satisfies $\pi\left(f^{\prime}\right)=\pi(f)=g$ because $\pi\left(f_{i_{0}}\right)=0$. If we write $f^{\prime}=\sum_{i=1}^{N} n_{i}^{\prime} e_{i}$ for $n_{i}^{\prime} \in \mathbb{Z}$ then we have $n_{i}^{\prime} \geq n_{i}$ for all $i$ and $n_{i_{0}}^{\prime} \geq 0$. Repeating this argument, we get an element $f \in \mathbb{N}^{N} \subset \mathbb{Z}^{N}$ which is fixed by the action of $\Gamma$ and satisfies $\pi(f)=g$. We are done.

Theorem 3.3. Let $\Gamma$ be a finite group, and $\mathcal{A}$ be a Kirchberg algebra. An action $\Gamma \curvearrowright$ $K_{*}(\mathcal{A})$ lifts to an action $\Gamma \curvearrowright \mathcal{A}$ if the induced two $\Gamma$-modules $K_{0}(\mathcal{A})$ and $K_{1}(\mathcal{A})$ have countable permutation presentations.

Proof. With the help of Theorem 1.8, it suffices to find

- a stable Kirchberg algebra $\mathcal{A}_{s}$ with an action of $\Gamma$ such that $K_{*}\left(\mathcal{A}_{s}\right) \cong\left(G_{0}, G_{1}\right)$ $\Gamma$-equivariantly, and

- a unital Kirchberg algebra $\mathcal{A}_{u}$ with an action of $\Gamma$ such that $K_{*}\left(\mathcal{A}_{u}\right) \cong\left(G_{0}, g, G_{1}\right)$ $\Gamma$-equivariantly

for $\Gamma$-modules $G_{0}, G_{1}$ having countable permutation presentations and $g \in G_{0}$ fixed by the action of $\Gamma$.

Take $\Gamma$-modules $G_{0}, G_{1}$ having countable permutation presentations. Let $N \in \mathfrak{C}$, an action $\Gamma \curvearrowright\{1,2, \ldots, N\}$ and $A, B \in M_{N}(\mathbb{Z})^{\Gamma}$ be as in the conclusion of Proposition 3.2. Set $\mathcal{A}:=\mathcal{O}_{A, B}$ which is a Kirchberg algebra by Proposition 2.10. Since $A, B \in M_{N}(\mathbb{Z})^{\Gamma}$ we have an action $\Gamma \curvearrowright \mathcal{A}$, and by Corollary 2.7 we get $K_{i}(\mathcal{A}) \cong G_{i}$ as $\Gamma$-modules for $i=0,1$. Let $\mathcal{K}$ be the $C^{*}$-algebra of all compact operators on the separable infinite dimensional Hilbert space $\ell^{2}(\mathbb{N})$. We define $\mathcal{A}_{s}:=\mathcal{A} \otimes \mathcal{K}$ which is a stable Kirchberg algebra. The action $\Gamma \curvearrowright \mathcal{A}$ extends to an action $\Gamma \curvearrowright \mathcal{A}_{s}$ by acting $\mathcal{K}$ trivially, and we have a $\Gamma$-equivariant isomorphism $K_{*}\left(\mathcal{A}_{s}\right) \cong\left(G_{0}, G_{1}\right)$.

Now take an element $g \in G_{0}$ fixed by the action of $\Gamma$. By Proposition 3.2, there exists $f \in \mathbb{N}^{N} \subset \mathbb{Z}^{N}$ fixed by the action of $\Gamma$ such that $[f] \in \operatorname{coker}(I-A)$ corresponds to $g \in G_{0}$. Let us denote $f=\sum_{i=1}^{N} n_{i} e_{i}$ with $n_{i} \in \mathbb{N}$. For each $n \in \mathbb{N}$, choose a projection $q_{n} \in \mathcal{K}$ whose rank is $n$. Let us define $p=\sum_{i=1}^{N} p_{i} \otimes q_{n_{i}} \in \mathcal{A} \otimes \mathcal{K}=\mathcal{A}_{s}$. Since $f \in \mathbb{Z}^{N}$ is fixed by the action of $\Gamma$, the projection $p \in \mathcal{A}_{s}$ is also fixed by the action of $\Gamma$. Hence the action $\Gamma \curvearrowright \mathcal{A}_{s}$ globally fixes the unital Kirchberg algebra $\mathcal{A}_{u}:=p \mathcal{A}_{s} p$. By Proposition 2.6, the element $[p] \in K_{0}\left(\mathcal{A}_{s}\right)$ corresponds to $[f] \in \operatorname{coker}(I-A)$ and hence to $g \in G_{0}$ by the isomorphisms $K_{0}\left(\mathcal{A}_{s}\right) \cong \operatorname{coker}(I-A) \cong G_{0}$. Hence we have $K_{*}\left(\mathcal{A}_{u}\right) \cong\left(G_{0}, g, G_{1}\right)$ $\Gamma$-equivariantly. We are done.

Remark 3.4. It seems to be possible to prove the theorem above using the construction in $[\mathrm{Sp}]$.

Theorem 3.5. Let $\Gamma$ be a finite group all of whose Sylow subgroups are cyclic and $\mathcal{A}$ be a Kirchberg algebra. Then every action $\Gamma \curvearrowright K_{*}(\mathcal{A})$ lifts to an action $\Gamma \curvearrowright \mathcal{A}$.

Proof. Combine Theorem 1.6 and Theorem 3.3.

Corollary 3.6. Let $\mathcal{A}$ be a Kirchberg algebra. Then every automorphism of $K_{*}(\mathcal{A})$ can be lifted to an automorphism of $\mathcal{A}$ with same order. 
Proof. For an automorphism of $K_{*}(\mathcal{A})$ with infinite order, this follows from [Ki, Ph]. For an automorphism of $K_{*}(\mathcal{A})$ with finite order $n$, this follows from Theorem 3.5 because a finite cyclic group $\mathbb{Z} / n \mathbb{Z}$ satisfies the assumption of Theorem 3.5.

We are going to see some examples to which we can or cannot apply Theorem 3.3 or Theorem 3.5. A unital Kirchberg algebra $\mathcal{A}$ is said to be in the Cuntz standard form if $\left[1_{\mathcal{A}}\right]=0$ in $K_{0}(\mathcal{A})$. For $n=2,3, \ldots, \infty$, we denote by $\mathcal{O}_{n}^{\text {st }}$ the unital Kirchberg algebra in the Cuntz standard form with isomorphic $K$-groups as the Cuntz algebra $\mathcal{O}_{n}$. Thus we have $K_{*}\left(\mathcal{O}_{n+1}^{\text {st }}\right)=(\mathbb{Z} / n \mathbb{Z}, 0,0)$ for $n<\infty$ and $K_{*}\left(\mathcal{O}_{\infty}^{\text {st }}\right)=(\mathbb{Z}, 0,0)$. Note that $\mathcal{O}_{n+1}^{\text {st }} \cong M_{n}\left(\mathcal{O}_{n+1}\right)$ for $n<\infty$.

Example 3.7. Let a finite group $\Gamma_{1} \cong \mathbb{Z} / 2 \mathbb{Z}$ and a $\Gamma_{1}$-module $G=\mathbb{Z} / 3 \mathbb{Z}$ be as in Example 1.3. In this example, we construct a unital Kirchberg algebras $\mathcal{A}$ and an action $\Gamma_{1} \curvearrowright \mathcal{A}$ such that $K_{0}(\mathcal{A}) \cong G$ as $\Gamma_{1}$-modules and $K_{1}(\mathcal{A})=0$ using the countable permutation presentation in Example 1.3. Note that $0 \in G$ is the only element which is fixed by the action of $\Gamma_{1}$.

Let us define an action $\Gamma_{1} \curvearrowright\{1,2,3,4\}$ by $\sigma(1)=2, \sigma(2)=1, \sigma(3)=4$ and $\sigma(4)=3$. Let $A, B \in M_{4}(\mathbb{Z})^{\Gamma_{1}}$ be

$$
A=\left(\begin{array}{llll}
2 & 0 & 3 & 0 \\
0 & 2 & 0 & 3 \\
1 & 0 & 2 & 1 \\
0 & 1 & 1 & 2
\end{array}\right), \quad B=\left(\begin{array}{llll}
1 & 0 & 1 & 0 \\
0 & 1 & 0 & 1 \\
1 & 0 & 1 & 0 \\
0 & 1 & 0 & 1
\end{array}\right) .
$$

These two matrices are obtained by applying the argument in the proof of Lemma 3.1 for $A^{\prime}, B^{\prime}, Y \in M_{2}(\mathbb{Z})^{\Gamma_{1}}$ defined by

$$
A^{\prime}=\left(\begin{array}{cc}
2 & -1 \\
-1 & 2
\end{array}\right), \quad B^{\prime}=\left(\begin{array}{ll}
1 & 0 \\
0 & 1
\end{array}\right), \quad Y=\left(\begin{array}{ll}
1 & 1 \\
1 & 1
\end{array}\right) .
$$

Although this $Y$ does not coincide with the one obtained from $A^{\prime}, B^{\prime}$ as in the proof of Lemma 3.1, we can still show that $A, B$ satisfies the conditions (0), (1), (2), and we have $\operatorname{ker}(I-A)=\operatorname{ker}(I-B)=\operatorname{coker}(I-B)=0$ and $\operatorname{coker}(I-A) \cong G$ as $\Gamma_{1}$-modules. Set $\mathcal{A}:=\mathcal{O}_{A, B}$ which is a unital Kirchberg algebra. Since $A, B \in M_{4}(\mathbb{Z})^{\Gamma_{1}}$, we get an action $\Gamma_{1} \curvearrowright \mathcal{A}$ and we have $K_{0}(\mathcal{A}) \cong G$ as $\Gamma_{1}$-modules and $K_{1}(\mathcal{A})=0$. By Theorem $1.8, \mathcal{A}$ is isomorphic to $\mathcal{O}_{4}^{\text {st }}$.

Example 3.8. Similarly as in the previous example, we see that the $C^{*}$-algebra $\mathcal{A}=\mathcal{O}_{A, B}$ is a unital Kirchberg algebra with an action $\Gamma_{1} \curvearrowright \mathcal{A}$ such that $K_{0}(\mathcal{A}) \cong K_{1}(\mathcal{A}) \cong G$ as $\Gamma_{1}$-modules where an action $\Gamma_{1} \curvearrowright\{1,2,3,4\}$ is same as in the previous example and $A, B \in M_{4}(\mathbb{Z})^{\Gamma_{1}}$ is defined by

$$
A=\left(\begin{array}{llll}
2 & 0 & 3 & 1 \\
0 & 2 & 1 & 3 \\
1 & 0 & 2 & 2 \\
0 & 1 & 2 & 2
\end{array}\right), \quad B=\left(\begin{array}{cccc}
1 & 0 & 2 & -1 \\
0 & 1 & -1 & 2 \\
1 & 0 & 1 & 0 \\
0 & 1 & 0 & 1
\end{array}\right) .
$$

By Theorem 1.8, $\mathcal{A}$ is isomorphic to $\mathcal{O}_{4}^{\text {st }} \otimes \mathcal{O}_{4}^{\text {st }}$.

Example 3.9. Let a finite group $\Gamma_{2} \cong(\mathbb{Z} / 2 \mathbb{Z}) \times(\mathbb{Z} / 2 \mathbb{Z})$ and a $\Gamma_{2}$-module $G=(\mathbb{Z} / 3 \mathbb{Z})^{3}$ be as in Example 1.4. We construct a unital Kirchberg algebras $\mathcal{A}$ and an action $\Gamma_{2} \curvearrowright \mathcal{A}$ such that $K_{0}(\mathcal{A}) \cong G$ as $\Gamma_{2}$-modules and $K_{1}(\mathcal{A})=0$. Note that $0 \in G$ is the only element fixed by the action of $\Gamma_{2}$. 
We define an action $\Gamma_{2} \curvearrowright\{1,2, \ldots, 8\}$ by

$$
\begin{aligned}
& \sigma: 1 \mapsto 2, \quad 2 \mapsto 1, \quad 3 \mapsto 4, \quad 4 \mapsto 3, \quad 5 \mapsto 6, \quad 6 \mapsto 5, \quad 7 \mapsto 8, \quad 8 \mapsto 7, \\
& \tau: 1 \mapsto 3, \quad 2 \mapsto 4, \quad 3 \mapsto 1, \quad 4 \mapsto 2, \quad 5 \mapsto 7, \quad 6 \mapsto 8, \quad 7 \mapsto 5, \quad 8 \mapsto 6 .
\end{aligned}
$$

We set $A, B \in M_{8}(\mathbb{Z})^{\Gamma_{2}}$ by

$$
A=\left(\begin{array}{llllllll}
2 & 0 & 0 & 0 & 4 & 0 & 0 & 0 \\
0 & 2 & 0 & 0 & 0 & 4 & 0 & 0 \\
0 & 0 & 2 & 0 & 0 & 0 & 4 & 0 \\
0 & 0 & 0 & 2 & 0 & 0 & 0 & 4 \\
1 & 0 & 0 & 0 & 2 & 1 & 1 & 1 \\
0 & 1 & 0 & 0 & 1 & 2 & 1 & 1 \\
0 & 0 & 1 & 0 & 1 & 1 & 2 & 1 \\
0 & 0 & 0 & 1 & 1 & 1 & 1 & 2
\end{array}\right), \quad B=\left(\begin{array}{llllllll}
1 & 0 & 0 & 0 & 1 & 0 & 0 & 0 \\
0 & 1 & 0 & 0 & 0 & 1 & 0 & 0 \\
0 & 0 & 1 & 0 & 0 & 0 & 1 & 0 \\
0 & 0 & 0 & 1 & 0 & 0 & 0 & 1 \\
1 & 0 & 0 & 0 & 1 & 0 & 0 & 0 \\
0 & 1 & 0 & 0 & 0 & 1 & 0 & 0 \\
0 & 0 & 1 & 0 & 0 & 0 & 1 & 0 \\
0 & 0 & 0 & 1 & 0 & 0 & 0 & 1
\end{array}\right) .
$$

Then $\mathcal{A}:=\mathcal{O}_{A, B}$ is a unital Kirchberg algebra, and the action $\Gamma_{2} \curvearrowright\{1,2, \ldots, 8\}$ defines an action $\Gamma_{2} \curvearrowright \mathcal{A}$ so that $K_{0}(\mathcal{A}) \cong G$ as $\Gamma_{2}$-modules and $K_{1}(\mathcal{A})=0$.

We have $\Gamma_{A, B} \cong \mathfrak{S}_{4}$ where $\mathfrak{S}_{4}:=\operatorname{Aut}(\{1,2,3,4\})$. Thus we get an action $\mathfrak{S}_{4} \curvearrowright \mathcal{A}$ such that the action $\Gamma_{2} \curvearrowright \mathcal{A}$ is its restriction. The induced action $\mathfrak{S}_{4} \curvearrowright K_{0}(\mathcal{A}) \cong(\mathbb{Z} / 3 \mathbb{Z})^{3}$ coincides with the natural action of $\mathfrak{S}_{4}$ on

$$
\left\{\left(a_{1}, a_{2}, a_{3}, a_{4}\right) \in(\mathbb{Z} / 3 \mathbb{Z})^{4} \mid a_{1}+a_{2}+a_{3}+a_{4}=0\right\} \cong(\mathbb{Z} / 3 \mathbb{Z})^{3} .
$$

Note that the group $\Gamma_{2}$ as well as $\mathfrak{S}_{4}$ does not satisfy the assumption of Theorem 3.5. Note also that the $\mathfrak{S}_{4}$-module $(\mathbb{Z} / 3 \mathbb{Z})^{3}$ has a countable permutation presentation as we can see from the matrix $A$ above or the matrix $D$ in Example 1.4.

Example 3.10. The author does not know how to construct a Kirchberg algebra $\mathcal{A}$ and an action $\Gamma_{2} \curvearrowright \mathcal{A}$ such that $K_{0}(\mathcal{A})$ is isomorphic to the $\Gamma_{2}$-module $G^{\prime}=(\mathbb{Z} / 4 \mathbb{Z})^{3}$ in Example 1.5.

Example 3.11. Let $\Gamma_{2} \cong(\mathbb{Z} / 2 \mathbb{Z}) \times(\mathbb{Z} / 2 \mathbb{Z})$ be as in the two previous examples. Let $G=\mathbb{Z} / 8 \mathbb{Z}$ be a $\Gamma_{2}$-module where an action of $\Gamma_{2}$ on $G$ is defined by $\sigma(g)=-g$ and $\tau(g)=3 g$. In [Ka5, Example 2.7], we saw that the $\Gamma_{2}$-module $G$ has no countable permutation presentations. Thus we cannot apply Theorem 3.3 nor Theorem 3.5. However Lemma 3.12 below together with Theorem 3.5 shows that there exists a unital Kirchberg algebra $\mathcal{A}$ in the Cuntz standard form and an action $\Gamma_{2} \curvearrowright \mathcal{A}$ such that $K_{0}(\mathcal{A}) \cong G$ as $\Gamma_{2}$-modules and $K_{1}(\mathcal{A})=0$. By Theorem 1.8 , we see that $\mathcal{A} \cong \mathcal{O}_{9}^{\text {st }}$. This example shows that neither conditions in Theorem 3.3 nor Theorem 3.5 is necessary.

Lemma 3.12. Let $\Gamma$ be a finite group, and $\mathcal{A}$ be a unital Kirchberg algebra in the Cuntz standard form. Suppose that $\Gamma$ decomposes as a product $\Gamma=\Gamma_{1} \times \Gamma^{\prime}$ where $\Gamma_{1}$ and $\Gamma^{\prime}$ are subgroups of $\Gamma$ such that $\Gamma_{1}=\{1, \sigma\} \cong \mathbb{Z} / 2 \mathbb{Z}$. Then an action $\Gamma \curvearrowright K_{*}(\mathcal{A})$ lifts to an action $\Gamma \curvearrowright \mathcal{A}$ if the restricted action $\Gamma^{\prime} \curvearrowright K_{*}(\mathcal{A})$ lifts to an action $\Gamma^{\prime} \curvearrowright \mathcal{A}$ and $\sigma(x)=-x$ for all $x \in K_{i}(\mathcal{A})$ with $i=0,1$.

Proof. By Theorem 3.5, there exists an action $\Gamma_{1} \curvearrowright \mathcal{O}_{\infty}^{\text {st }}$ such that the induced action $\Gamma_{1} \curvearrowright K_{*}\left(\mathcal{O}_{\infty}^{\text {st }}\right)$ satisfies $\sigma(x)=-x$ for $x \in K_{0}\left(\mathcal{O}_{\infty}^{\text {st }}\right) \cong \mathbb{Z}$. Then the tensor product action $\Gamma=\Gamma_{1} \times \Gamma^{\prime} \curvearrowright \mathcal{O}_{\infty}^{\text {st }} \otimes \mathcal{A} \cong \mathcal{A}$ is a lifting of the given action $\Gamma \curvearrowright K_{*}(\mathcal{A})$ whose restriction to $\Gamma^{\prime}$ lifts to $\Gamma^{\prime} \curvearrowright \mathcal{A}$ and which satisfies $\sigma(x)=-x$ for all $x \in K_{i}(\mathcal{A})$ with $i=0,1$. 
Remark 3.13. A similar statement as this lemma holds for stable Kirchberg algebras. However it seems that the argument above does not work for unital Kirchberg algebras which is not in the Cuntz standard form. See the next example.

Example 3.14. The author does not know how to construct a unital Kirchberg algebra $\mathcal{A}$ and an action $\Gamma_{2} \curvearrowright \mathcal{A}$ such that $K_{0}(\mathcal{A})$ is isomorphic to $\Gamma_{2}$-module $G$ in Example 3.11 and $\left[1_{\mathcal{A}}\right] \in K_{0}(\mathcal{A})$ corresponds to $4 \in G$ which is fixed by the action of $\Gamma_{2}$. Thus it seems that in practice we need to worry about the position $\left[1_{\mathcal{A}}\right] \in K_{0}(\mathcal{A})$ when considering the lifting problem.

We finish this section by considering the splitting problem stated in the introduction of this paper. The following is easy to see.

Proposition 3.15. For a Kirchberg algebra $\mathcal{A}$ which has the isomorphic K-groups as $\mathcal{O}_{\infty}$, the surjection $\operatorname{Aut}(\mathcal{A}) \rightarrow \operatorname{Aut}\left(K_{*}(\mathcal{A})\right)$ splits.

Proof. Such a Kirchberg algebra $\mathcal{A}$ is isomorphic to $M_{k}\left(\mathcal{O}_{\infty}\right)$ for a positive integer $k, \mathcal{O}_{\infty}^{\text {st }}$ or $\mathcal{O}_{\infty} \otimes \mathcal{K}$ where $\mathcal{K}$ is the $C^{*}$-algebra of all compact operators on $\ell^{2}(\mathbb{N})$. For $\mathcal{A}=M_{k}\left(\mathcal{O}_{\infty}\right)$, we have $K_{*}(\mathcal{A})=(\mathbb{Z}, k, 0)$. Hence $\operatorname{Aut}\left(K_{*}(\mathcal{A})\right)$ is trivial, and we need to do nothing. For $\mathcal{A}=\mathcal{O}_{\infty}^{\text {st }}$ or $\mathcal{A}=\mathcal{O}_{\infty} \otimes \mathcal{K}$, we have $\operatorname{Aut}\left(K_{*}(\mathcal{A})\right) \cong \mathbb{Z} / 2 \mathbb{Z}$. Hence by Theorem 3.5 the surjection $\operatorname{Aut}(\mathcal{A}) \rightarrow \operatorname{Aut}\left(K_{*}(\mathcal{A})\right)$ splits.

For a Cuntz algebra $\mathcal{O}_{n+1}$ with $n=1,2, \ldots$, the group $\operatorname{Aut}\left(K_{*}\left(\mathcal{O}_{n+1}\right)\right)$ becomes trivial. Thus we need to do nothing. For a Kirchberg algebra $\mathcal{O}_{n+1}^{\text {st }}$ in the Cuntz standard form with $n=1,2, \ldots$, we have $\operatorname{Aut}\left(K_{*}\left(\mathcal{O}_{n+1}^{\text {st }}\right)\right) \cong(\mathbb{Z} / n \mathbb{Z})^{\times}$. On the splitting problem for these Kirchberg algebras, we get the following:

Proposition 3.16. The surjection $\operatorname{Aut}\left(\mathcal{O}_{n+1}^{s t}\right) \rightarrow \operatorname{Aut}\left(K_{*}\left(\mathcal{O}_{n+1}^{s t}\right)\right)$ splits if $(\mathbb{Z} / n \mathbb{Z})^{\times}$is a cyclic group or a product of $\mathbb{Z} / 2 \mathbb{Z}$ and a cyclic group.

Proof. When $(\mathbb{Z} / n \mathbb{Z})^{\times}$is a cyclic group, this follows from Theorem 3.5. When $(\mathbb{Z} / n \mathbb{Z})^{\times}$ is a product of $\mathbb{Z} / 2 \mathbb{Z}$ and a cyclic group, this follows from Theorem 3.5 and Lemma 3.12 because in this case we can take $-1 \in(\mathbb{Z} / n \mathbb{Z})^{\times}$as the generator of $\mathbb{Z} / 2 \mathbb{Z} \subset(\mathbb{Z} / n \mathbb{Z})^{\times}$(see the list below).

Note that a finite abelian group satisfies the assumption of Theorem 3.5 if and only if it is cyclic. The following is the complete list of positive integers $n$ satisfying the assumption in Proposition 3.16:

- $(\mathbb{Z} / n \mathbb{Z})^{\times}$is cyclic if and only if

- $n=p^{l}$ for an odd prime number $p$ and a positive integer $l$ or

- $n=2 p^{l}$ for an odd prime number $p$ and a positive integer $l$ or

$\cdot n=1,2,4$.

- $(\mathbb{Z} / n \mathbb{Z})^{\times} \cong(\mathbb{Z} / 2 \mathbb{Z}) \times(\mathbb{Z} / 2 m \mathbb{Z})$ for a positive integer $m$ if and only if

- $n=p_{1}^{l_{1}} p_{2}^{l_{2}}$ for $p_{1}, p_{2}, l_{1}, l_{2}$ satisfying $(*)$ below or

- $n=2 p_{1}^{l_{1}} p_{2}^{l_{2}}$ for $p_{1}, p_{2}, l_{1}, l_{2}$ satisfying $(*)$ below or

- $n=4 p^{l}$ for an odd prime number $p$ and a positive integer $l$ or

- $n=2^{l}$ for $l=3,4, \ldots$.

(*) $\begin{aligned} & p_{1}, p_{2} \text { are distinct odd prime numbers } \\ & \text { and } l_{1}, l_{2} \text { are positive integers such that }\end{aligned}\left\{\begin{array}{l}\cdot\left(p_{1}-1, p_{2}-1\right)=2 \text { and } \\ \cdot\left(p_{1}, p_{2}-1\right)=1 \text { if } l_{1} \geq 2 \text { and } \\ \cdot\left(p_{1}-1, p_{2}\right)=1 \text { if } l_{2} \geq 2 .\end{array}\right.$ 
Positive integers $n$ which do not satisfy the assumption in Proposition 3.16 are

$$
n=24,40,48,56,60,63,65,72,80,84,85,88,91,96,104,105,112,117,120, \ldots
$$

It is clear that a similar statement of Proposition 3.16 holds for stable Kirchberg algebras $\mathcal{O}_{n+1} \otimes \mathcal{K}$. For a unital Kirchberg algebras $M_{k}\left(\mathcal{O}_{n+1}\right)$ which is not in the Cuntz standard form, the author does not know the answer of the splitting problem except the case Aut $\left(K_{*}\left(M_{k}\left(\mathcal{O}_{n+1}\right)\right)\right)$ is cyclic in which Theorem 3.5 gives an affirmative answer. In particular, he does not know the answer for $M_{4}\left(\mathcal{O}_{9}\right)$ (Example 3.14). However the following proposition says that in generic cases Aut $\left(K_{*}\left(M_{k}\left(\mathcal{O}_{n+1}\right)\right)\right)$ is cyclic and hence the splitting problem has an affirmative answer by Theorem 3.5. We omit the routine proof.

Proposition 3.17. Let $n$ and $k$ be positive integers. Let us write $n=2^{l_{0}} p_{1}^{l_{1}} p_{2}^{l_{2}} \cdots p_{m}^{l_{m}}$ for $l_{0} \in \mathbb{N}$, positive integers $l_{1}, \ldots, l_{m}$ and distinct odd prime numbers $p_{1}, \ldots, p_{m}$. Suppose that $k$ is not divided by $p_{i}^{l_{i}}$ for $i=1,2, \ldots, m$, and not divided by $2^{l_{0}-1}$ when $l_{0} \geq 3$. Then

$$
\operatorname{Aut}\left(K_{*}\left(M_{k}\left(\mathcal{O}_{n+1}\right)\right)\right)=\operatorname{Aut}((\mathbb{Z} / n \mathbb{Z}, k, 0)) \cong\left\{x \in(\mathbb{Z} / n \mathbb{Z})^{\times} \mid x k=k\right\}
$$

is a cyclic group, and hence the surjection $\operatorname{Aut}\left(M_{k}\left(\mathcal{O}_{n+1}\right)\right) \rightarrow \operatorname{Aut}\left(K_{*}\left(M_{k}\left(\mathcal{O}_{n+1}\right)\right)\right)$ splits by Theorem 3.5.

Remark 3.18. In [I, Subsection 6.3], Izumi discussed a problem which is similar to the splitting problem in this paper. His problem is equivalent to our splitting problem for a Kirchberg algebra $\mathcal{A}$ with $K_{i}(\mathcal{A})=0$ for $i=0$ or 1 . By Proposition 3.16, the surjection $\operatorname{Aut}\left(\mathcal{O}_{n+1}^{\text {st }}\right) \rightarrow \operatorname{Aut}\left(K_{*}\left(\mathcal{O}_{n+1}^{\text {st }}\right)\right)$ splits if $n$ is a power of a prime number. This extends the latter statement of [I, Theorem 6.12] and the result explained in [I, Remark 6.13]. The former statement of [I, Theorem 6.12] can also be extended by the following corollary of Theorem 3.3.

Corollary 3.19. Let $\Gamma$ be a finite group, and $\mathcal{A}$ be a Kirchberg algebra. An action $\Gamma \curvearrowright$ $K_{*}(\mathcal{A})$ lifts to an action $\Gamma \curvearrowright \mathcal{A}$ if the induced two $\Gamma$-modules $K_{0}(\mathcal{A})$ and $K_{1}(\mathcal{A})$ are cohomologically trivial.

Proof. By [Br, Theorem VI.8.12] and [Ka5, Proposition 3.1], every countable cohomologically trivial module has a countable permutation presentation. Hence Theorem 3.3 shows the conclusion.

\section{Relations to other Classes of $C^{*}$-Algebras}

4.1. Cuntz-Krieger algebras. In this subsection, we see relations between our $C^{*}$-algebras $\mathcal{O}_{A, B}$ and the Cuntz-Krieger algebras $\mathcal{O}_{A}$ introduced in $[\mathrm{CK}]$ and generalized in [EL1, KPR] and other papers. Recall that the Cuntz-Krieger algebra $\mathcal{O}_{A}$ of a matrix $A \in M_{N}(\mathbb{N})$ is the universal $C^{*}$-algebra generated by mutually orthogonal projections $\left\{p_{i}\right\}_{i=1}^{N}$ and partial isometries $\left\{s(n)_{i, j}\right\}_{(i, j) \in \Omega_{A}, n \in\left\{1,2, \ldots, A_{i, j}\right\}}$ satisfying the relations (ii) and (iii) in Definition 2.2. When $N=\infty, \mathcal{O}_{A}$ should be called the Exel-Laca algebra or the graph algebra of the row-finite graph associated with $A$ (see [EL1] or [Ra, Chapter 2]). Take $N \in \mathfrak{C}$ and $A, B \in M_{N}(\mathbb{Z})$ satisfying the condition (0), and fix them. We will show that the natural map $\mathcal{O}_{A} \rightarrow \mathcal{O}_{A, B}$ is injective. To this end, we need the following definition and proposition.

Definition 4.1. For each $z \in \mathbb{T}$ the universality of $\mathcal{O}_{A, B}$ shows the existence of an automorphism $\beta_{z}$ of $\mathcal{O}_{A, B}$ such that $\beta_{z}\left(p_{i}\right)=p_{i}, \beta_{z}\left(u_{i}\right)=u_{i}$ for each $i$ and $\beta_{z}\left(s(n)_{i, j}\right)=$ 
$z s(n)_{i, j}$ for each $(i, j) \in \Omega_{A}$ and $n \in \mathbb{Z}$. We call the action $\beta: \mathbb{T} \curvearrowright \mathcal{O}_{A, B}$ the gauge action of $\mathcal{O}_{A, B}$.

Proposition 4.2. For each $i \in\{1,2, \ldots, N\}$, the projection $p_{i} \in \mathcal{O}_{A, B}$ is non-zero.

Proof. It suffices to find operators $\left\{P_{i}\right\}_{i=1}^{N},\left\{U_{i}\right\}_{i=1}^{N}$, and $\left\{S(n)_{i, j}\right\}_{(i, j) \in \Omega_{A}, n \in \mathbb{Z}}$ on some Hilbert space such that they satisfy the relations (i) to (iii) in Definition 2.2 and $P_{i} \neq 0$ for all $i$.

We set

$$
\Omega_{A}^{\infty}:=\left\{\mu=\left(i_{0}, i_{1}, \ldots\right) \in\{1,2, \ldots, N\}^{\infty} \mid\left(i_{l-1}, i_{l}\right) \in \Omega_{A} \text { for } l=1,2, \ldots\right\} .
$$

For each $\mu=\left(i_{0}, i_{1}, \ldots\right) \in \Omega_{A}^{\infty}$, we define a group $F_{\mu}$ to be the abelian group generated by $\left\{\xi_{\mu}^{l}\right\}_{l=1}^{\infty}$ with relations $A_{i_{l-1}, i_{l}} \xi_{\mu}^{l}=B_{i_{l}, i_{l+1}} \xi_{\mu}^{l+1}$ for $l=1,2, \ldots$ For $(i, j) \in \Omega_{A}$ and $\mu=\left(i_{0}, i_{1}, \ldots\right) \in \Omega_{A}^{\infty}$ with $i_{0}=j$, we set $i \mu \in \Omega_{A}^{\infty}$ by $i \mu=\left(i, i_{0}, i_{1}, \ldots\right)$. We define a homomorphism $\sigma_{i}: F_{\mu} \rightarrow F_{i \mu}$ by $\sigma_{i}\left(\xi_{\mu}^{l}\right)=\xi_{i \mu}^{l+1}$ for all $l=1,2, \ldots$ It is easy to see that $\sigma_{i}$ is a well-defined injective homomorphism. It is also easy to see that we have $\sigma_{i}\left(B_{i_{0}, i_{1}} \xi_{\mu}^{1}\right)=A_{i, j} \xi_{i \mu}^{1}$, and the quotient $F_{i \mu} / \sigma_{i}\left(F_{\mu}\right)$ is isomorphic to $\mathbb{Z} / A_{i, j} \mathbb{Z}$ which is generated by the image of $\xi_{i \mu}^{1} \in F_{i \mu}$. Hence we have

$$
F_{i \mu}=\coprod_{n=1}^{A_{i, j}}\left(\sigma_{i}\left(F_{\mu}\right)+n \xi_{i \mu}^{1}\right) .
$$

Let $\mathcal{F}^{\infty}:=\coprod_{\mu \in \Omega_{A}^{\infty}} F_{\mu}$. We define operators $\left\{P_{i}\right\}_{i=1}^{N},\left\{U_{i}\right\}_{i=1}^{N}$, and $\left\{S(n)_{i, j}\right\}_{(i, j) \in \Omega_{A}, n \in \mathbb{Z}}$ on the Hilbert space $\ell^{2}\left(\mathcal{F}^{\infty}\right)$ by

$$
\begin{gathered}
P_{i}\left(\delta_{\xi}\right):=\left\{\begin{array}{ll}
\delta_{\xi} & \text { if } i_{0}=i \\
0 & \text { if } i_{0} \neq i,
\end{array} \quad U_{i}\left(\delta_{\xi}\right):= \begin{cases}\delta_{\xi+B_{i_{0}, i_{1}} \xi_{\mu}^{1}} & \text { if } i_{0}=i \\
0 & \text { if } i_{0} \neq i,\end{cases} \right. \\
S(n)_{i, j}\left(\delta_{\xi}\right):= \begin{cases}\delta_{\sigma_{i}(\xi)+n \xi_{i \mu}^{1}} & \text { if } i_{0}=j \\
0 & \text { if } i_{0} \neq j,\end{cases}
\end{gathered}
$$

for $\xi \in F_{\mu} \subset \mathcal{F}^{\infty}$ with $\mu=\left(i_{0}, i_{1}, \ldots\right) \in \Omega_{A}^{\infty}$. Then it is routine to check that these operators satisfy the relations (i) to (iii) in Definition 2.2. For each $i \in\{1,2, \ldots, N\}$ we can find $\mu=\left(i_{0}, i_{1}, \ldots\right) \in \Omega_{A}^{\infty}$ with $i_{0}=i$ because $\Omega_{A}(i) \neq \emptyset$ for all $i$. Hence $P_{i} \neq 0$ for all $i$. We are done.

As a corollary of this proposition, we get the following. We say that $B \in M_{N}(\mathbb{Z})$ has a zero row if there exists $i \in\{1,2, \ldots, N\}$ with $B_{i, j}=0$ for all $j \in\{1,2, \ldots, N\}$. Otherwise we say that $B \in M_{N}(\mathbb{Z})$ has no zero rows.

Corollary 4.3. The spectrum of $u_{i} \in \mathcal{O}_{A, B}$ contains $\mathbb{T}$ for all $i \in\{1,2, \ldots, N\}$ if and only if $B$ has no zero rows.

Proof. If there exists $i \in\{1,2, \ldots, N\}$ with $B_{i, j}=0$ for all $j \in\{1,2, \ldots, N\}$, then we have

$$
u_{i}=u_{i} p_{i}=u_{i}\left(\sum_{j \in \Omega_{A}(i)} \sum_{n=1}^{A_{i, j}} s(n)_{i, j} s(n)_{i, j}^{*}\right)=\sum_{j \in \Omega_{A}(i)} \sum_{n=1}^{A_{i, j}} s(n)_{i, j} s(n)_{i, j}^{*}=p_{i} .
$$

Hence if the spectrum of $u_{i} \in \mathcal{O}_{A, B}$ contains $\mathbb{T}$ for all $i$, then $B$ has no zero rows. Conversely suppose that $B$ has no zero rows. Take $i \in\{1,2, \ldots, N\}$. To show that the spectrum of $u_{i} \in \mathcal{O}_{A, B}$ contains $\mathbb{T}$, it suffices to show that the spectrum of the operator $U_{i}$ 
on $\ell^{2}\left(\mathcal{F}^{\infty}\right)$ defined in the proof of Proposition 4.2 contains $\mathbb{T}$. Since $B$ has no zero rows, we can find $\mu=\left(i_{0}, i_{1}, \ldots\right) \in \Omega_{A}^{\infty}$ with $i_{0}=i$ and $B_{i_{l-1}, i_{l}} \neq 0$ for all $l$. Then the order of the element $\xi:=B_{i_{0}, i_{1}} \xi_{\mu}^{1}$ in the group $F_{\mu}$ is infinite. Hence the spectrum of $U_{i}$ contains $\mathbb{T}$ because $U_{i}$ acts as a bilateral shift on $\ell^{2}(\mathbb{Z} \xi) \cong \ell^{2}(\mathbb{Z})$. This completes the proof.

Remark 4.4. From the operators on $H:=\ell^{2}\left(\mathcal{F}^{\infty}\right)$ defined in the proof of Proposition 4.2, we get a representation $\pi: \mathcal{O}_{A, B} \rightarrow B(H)$. In general, the representation $\pi$ is not faithful. However by computing the spectrum of $u_{i}$ and $U_{i}$, we can show that the restriction of $\pi$ to the $C^{*}$-algebra generated by $\left\{u_{i}\right\}_{i=1}^{N}$ is injective. From this fact and a variant of gauge-invariant uniqueness theorems, we can show that the $*$-homomorphism

$$
\tilde{\pi}: \mathcal{O}_{A, B} \rightarrow C(\mathbb{T}, B(H)) \cong C(\mathbb{T}) \otimes B(H) \subset B\left(L^{2}(\mathbb{T}) \otimes H\right)
$$

defined by $\tilde{\pi}(x)(z)=\pi\left(\beta_{z}(x)\right)$ is injective. Thus we get an explicit faithful representation of $\mathcal{O}_{A, B}$. Note that a variant of Cuntz-Krieger uniqueness theorems shows that the representation $\pi$ itself is faithful when $A$ and $B$ are sufficiently complicated.

Proposition 4.5. The natural *-homomorphism $\mathcal{O}_{A} \rightarrow \mathcal{O}_{A, B}$ defined by $p_{i} \mapsto p_{i}$ and $s(n)_{i, j} \mapsto s(n)_{i, j}$ is injective.

Proof. This follows from the gauge-invariant uniqueness theorem of the Cuntz-Krieger algebras (see [Ra, Theorem 2.2]) with the help of the gauge action $\beta$ of $\mathcal{O}_{A, B}$ and Proposition 4.2 .

By this proposition, we can consider the Cuntz-Krieger algebra $\mathcal{O}_{A}$ as a $C^{*}$-subalgebra of $\mathcal{O}_{A, B}$.

It is routine to see that $\sum_{i=1}^{N} p_{i}$ converges to the unit of the multiplier algebra $\mathcal{M}\left(\mathcal{O}_{A, B}\right)$ of $\mathcal{O}_{A, B}$ in the strict topology (when $N<\infty, \sum_{i=1}^{N} p_{i}$ is the unit of $\mathcal{O}_{A, B}$ ). Hence $\sum_{i=1}^{N} u_{i}$ converges to a unitary $u$ of $\mathcal{M}\left(\mathcal{O}_{A, B}\right)$ in the strict topology. The unitary $u \in \mathcal{M}\left(\mathcal{O}_{A, B}\right)$ has the following commutation relations with the generators of $\mathcal{O}_{A} \subset \mathcal{O}_{A, B}$;

$$
u p_{i}=p_{i} u\left(=u_{i}\right), \quad u^{k} s(n)_{i, j}=s\left(n^{\prime}\right)_{i, j} u^{k^{\prime}}\left(=s(m)_{i, j}\right)
$$

for all $i \in\{1,2, \ldots, N\},(i, j) \in \Omega_{A}, n, n^{\prime} \in\left\{1,2, \ldots, A_{i, j}\right\}$ and $k, k^{\prime} \in \mathbb{Z}$ with $n+k B_{i, j}=$ $n^{\prime}+k^{\prime} A_{i, j}(=m)$. The following proposition says that the $C^{*}$-algebra $\mathcal{O}_{A, B}$ can be defined to be the universal $C^{*}$-algebra generated by the product of the Cuntz-Krieger $\mathcal{O}_{A} \subset \mathcal{O}_{A, B}$ and the unitary $u \in \mathcal{M}\left(\mathcal{O}_{A, B}\right)$ with the commutation relations above.

Proposition 4.6. For a non-degenerate representation $\mathcal{O}_{A} \rightarrow B(H)$ and a unitary $U \in$ $B(H)$ satisfying the commutation relations above, there exists a unique representation $\mathcal{O}_{A, B} \rightarrow B(H)$ extending the representation $\mathcal{O}_{A} \rightarrow B(H)$ and sending the unitary $u \in$ $\mathcal{M}\left(\mathcal{O}_{A, B}\right)$ to $U \in B(H)$.

Proof. Straightforward.

When $B=0$ or $B=A$, the $C^{*}$-algebra $\mathcal{O}_{A, B}$ can be explicitly described using the Cuntz-Krieger algebra $\mathcal{O}_{A}$.

Proposition 4.7. For a matrix $A \in M_{N}(\mathbb{N})$, we have $\mathcal{O}_{A, 0} \cong \mathcal{O}_{A}$ and $\mathcal{O}_{A, A} \cong \mathcal{O}_{A} \otimes C(\mathbb{T})$.

Proof. When $B=0$, we have $u_{i}=p_{i}$ for all $i$ as computed in the proof of Corollary 4.3. This shows that the $*$-homomorphism $\mathcal{O}_{A} \rightarrow \mathcal{O}_{A, 0}$ in Proposition 4.5 is surjective, and hence an isomorphism. Note that in this case we have $u=1$. 
When $B=A$, the unitary $u \in \mathcal{M}\left(\mathcal{O}_{A, A}\right)$ commutes with $\mathcal{O}_{A} \subset \mathcal{O}_{A, A}$. Hence $\mathcal{O}_{A, A} \cong$ $\mathcal{O}_{A} \otimes C(\mathbb{T})$ by Proposition 4.6.

4.2. Topological graph algebras. In [Ka1], a notion of topological graphs was introduced, and a construction of a $C^{*}$-algebra $\mathcal{O}(E)$ from a topological graph $E$ was given. This construction generalizes the one of Cuntz-Krieger algebras or more generally of graph algebras. In [Ka4, Definition 6.1] a topological graph $E_{A, B}$ was constructed from two matrices $A \in M_{\infty}(\mathbb{N})$ and $B \in M_{\infty}(\mathbb{Z})$ such that $A_{i, j}=0$ implies $B_{i, j}=0$, and in $\left[\mathrm{Ka} 4\right.$, Proposition B.1] generators and relations of the $C^{*}$-algebra $\mathcal{O}\left(E_{A, B}\right)$ were provided. The definition of the $C^{*}$-algebra $\mathcal{O}_{A, B}$ is motivated by these generators and relations of $\mathcal{O}\left(E_{A, B}\right)$, and in fact by [Ka4, Proposition B.2] we have $\mathcal{O}_{A, B} \cong \mathcal{O}\left(E_{A, B}\right)$ for $A, B \in M_{\infty}(\mathbb{Z})$ satisfying the condition (0) if $B$ has no zero rows. As pointed out in [Ka4, Remark 6.3], a similar construction of a topological graph $E_{A, B}$ from $A, B \in M_{N}(\mathbb{Z})$ satisfying the condition ( 0 ) for $N<\infty$ is possible, and we can show $\mathcal{O}_{A, B} \cong \mathcal{O}\left(E_{A, B}\right)$ if $B$ has no zero rows. However in the case that $B$ has a zero row, the natural surjection from $\mathcal{O}\left(E_{A, B}\right)$ to $\mathcal{O}_{A, B}$ is never injective because there exists a restriction " $v \in E_{\mathrm{rg}}^{0} \cap E_{m}^{0}$ " in the relation (iii) of [Ka4, Proposition B.2] although we do not consider the corresponding restriction when defining $\mathcal{O}_{A, B}$. This restriction looks natural from the graph algebraic point of view (cf. the fact that the Cuntz-Krieger relation (CK2) is not assumed at sources in p.6 of $[\mathrm{Ra}])$. We do not adopt this restriction because this seems to be unnatural from our point of view in this paper, and makes the computation of $K$-groups in Proposition 2.6 complicated.

In the case that $B$ has no zero rows, the computation of $K$-groups in Proposition 2.6 without considering $\Gamma_{A, B}$-actions follows from [Ka4, Lemma 6.2], and Proposition 2.9 follows from [Ka1, Propositions 6.1 and 6.6]. Even when $B$ has a zero row, we can get Proposition 2.9 using the theory of topological graph algebras with extra efforts. We give direct complete proofs of them in the following three sections without this restriction on $B$ because we naturally get it on the way to other results.

We can show Proposition 2.10 from [Ka4, Proposition 6.5] if $N=\infty$, and by repeating a similar argument we can show it for $N<\infty$. For the readers' convenience, we give a self-contained proof of Proposition 2.10 in Section 6 instead of forcing the readers follow long arguments from [Ka1] to [Ka4] which are too long and too general for our purpose.

4.3. Cuntz-Pimsner algebras. In [Pi], Pimsner introduced a construction of a $C^{*}$-algebra $\mathcal{O}_{\mathbf{X}}$ from a $C^{*}$-correspondence $\mathrm{X}$ which was called a Hilbert bimodule in [Pi]. This $C^{*}$-algebra $\mathcal{O}_{\mathbf{X}}$ is now called a Cuntz-Pimsner algebra, and it generalizes Cuntz-Krieger algebras. From $N \in \mathfrak{C}$ and two matrices $A, B \in M_{N}(\mathbb{Z})$ satisfying the condition (0), we can define a full $C^{*}$-correspondence $\mathrm{X}_{A, B}\left(\cong \bigoplus_{(i, j) \in \Omega_{A}} C(\mathbb{T})\right)$ over a commutative $C^{*}$-algebra $\mathcal{A}_{N} \cong C_{0}(\{1,2, \ldots, N\} \times \mathbb{T})$ in a similar way as in [Ka4], and show that the CuntzPimsner algebra $\mathcal{O}_{\mathbf{X}_{A, B}}$ is isomorphic to our $C^{*}$-algebra $\mathcal{O}_{A, B}$. We remark that in [Ka2] a modified construction of Cuntz-Pimsner algebras was proposed so that it generalizes topological graph algebras, but here we mean the original definition of Cuntz-Pimsner algebras. Hence the natural *-homomorphism $\mathcal{A}_{N} \rightarrow \mathcal{O}_{\mathrm{X}_{A, B}}$ is injective if and only if the left action of the $C^{*}$-correspondence $\mathrm{X}_{A, B}$ is faithful which turns out to be equivalent that $B$ has no zero rows (cf. Corollary 4.3). We also note that the Toeplitz algebra $\mathcal{T}_{\mathbf{X}_{A, B}}$ defined in $[\mathrm{Pi}]$ is isomorphic to the $C^{*}$-algebra $\mathcal{T}_{A, B}$ which will be defined in Definition 7.1, 
and that we have

$$
\begin{aligned}
\mathcal{A}_{N} & \cong \overline{\operatorname{span}}\left\{u_{i}^{n} \mid i \in\{1,2, \ldots, N\}, n \in \mathbb{Z}\right\} \subset \mathcal{T}_{A, B}, \\
\mathrm{X}_{A, B} & \cong \overline{\operatorname{span}}\left\{s(n)_{i, j} \mid(i, j) \in \Omega_{A}, n \in \mathbb{Z}\right\} \subset \mathcal{T}_{A, B} .
\end{aligned}
$$

In the proof of Proposition 2.6, we use the brilliant idea of Pimsner in [Pi, Section 4] for computing the $K$-groups of Cuntz-Pimsner algebras. We repeat Pimsner's argument in our terminology in Section 7 because the left actions of our $C^{*}$-correspondence $\mathrm{X}_{A, B}$ need not be faithful although it was assumed to be faithful in [Pi], and because we also have to compute $\Gamma_{A, B}$-actions on the $K$-groups of $\mathcal{O}_{A, B}$. When $B$ has no zero rows, Proposition 2.9 follows from the theory of Cuntz-Pimsner algebras (see [Ka3] for example).

\section{Structures and the nuclearity of $\mathcal{O}_{A, B}$}

Let $A, B \in M_{N}(\mathbb{Z})$ satisfy the condition (0). In this section, we define a dense $*$-algebra $\mathcal{O}_{A, B}^{\text {alg }}$ of $\mathcal{O}_{A, B}$ whose elements are described explicitly, and show that $\mathcal{O}_{A, B}$ is nuclear by examining the so-called core $\mathcal{O}_{A, B}^{\mathbb{T}}$ of $\mathcal{O}_{A, B}$. The analysis in this section will be used in the next section.

We set $\Omega_{A}^{0}:=\{(1),(2), \ldots,(N)\}$, and

$$
\Omega_{A}^{k}:=\left\{\mu=\left(i_{0}, i_{1}, \ldots, i_{k}\right) \in\{1,2, \ldots, N\}^{k+1} \mid\left(i_{l-1}, i_{l}\right) \in \Omega_{A} \text { for } l=1,2, \ldots, k\right\},
$$

for a positive integer $k$. We define $\Omega_{A}^{*}:=\bigsqcup_{k \in \mathbb{N}} \Omega_{A}^{k}$.

For $(i) \in \Omega_{A}^{0}$, let $F_{(i)} \cong \mathbb{Z}$ be the cyclic group whose generator is given by $\xi_{(i)}^{0} \in F_{(i)}$. For $\xi=n \xi_{(i)}^{0} \in F_{(i)}$ with $n \in \mathbb{Z}$, we define $s_{\xi}:=u_{i}^{n} \in \mathcal{O}_{A, B}$. Let us take $\mu=\left(i_{0}, i_{1}, \ldots, i_{k}\right) \in$ $\Omega_{A}^{k}$ for a positive integer $k$. Let $F_{\mu}$ be the abelian group generated by elements $\left\{\xi_{\mu}^{l}\right\}_{l=1}^{k}$ with relations $A_{i_{l-1}, i_{l}} \xi_{\mu}^{l}=B_{i_{l}, i_{l+1}} \xi_{\mu}^{l+1}$ for $l=1,2, \ldots, k-1$. For $\xi=\sum_{l=1}^{k} n_{l} \xi_{\mu}^{l} \in F_{\mu}$ with $n_{1}, n_{2}, \ldots, n_{k} \in \mathbb{Z}$, we define $s_{\xi} \in \mathcal{O}_{A, B}$ by

$$
s_{\xi}:=s\left(n_{1}\right)_{i_{0}, i_{1}} S\left(n_{2}\right)_{i_{1}, i_{2}} \cdots s\left(n_{k}\right)_{i_{k-1}, i_{k}} .
$$

It is routine to check that this is well-defined, and $s_{\xi}$ satisfies $s_{\xi}^{*} s_{\xi}=p_{i_{k}}$.

We set $\mathcal{F}^{k}:=\coprod_{\mu \in \Omega_{A}^{k}} F_{\mu}$ for $k \in \mathbb{N}$ and $\mathcal{F}^{*}:=\coprod_{\mu \in \Omega_{A}^{*}} F_{\mu}$. Thus $\mathcal{F}^{*}=\coprod_{k \in \mathbb{N}} \mathcal{F}^{k}$. We define

$$
\mathcal{O}_{A, B}^{\text {alg }}:=\operatorname{span}\left\{s_{\xi} s_{\eta}^{*} \mid \xi, \eta \in \mathcal{F}^{*}\right\} .
$$

By the following lemma, $\mathcal{O}_{A, B}^{\text {alg }}$ is the $*$-algebra generated by the generators $\left\{p_{i}, u_{i}, s(n)_{i, j}\right\}$, and hence dense in $\mathcal{O}_{A, B}$.

Lemma 5.1. For $\xi \in \mathcal{F}^{k}$ and $\eta \in \mathcal{F}^{l}$ with $k, l \in \mathbb{N}$, we have the following.

- We have either $s_{\xi} s_{\eta}=0$ or $s_{\xi} s_{\eta}=s_{\zeta}$ for some $\zeta \in \mathcal{F}^{k+l}$.

- When $k \geq l$, we have either $s_{\eta}^{*} s_{\xi}=0$ or $s_{\eta}^{*} s_{\xi}=s_{\zeta}$ for some $\zeta \in \mathcal{F}^{k-l}$.

- When $k=l \geq 1$, we have $s_{\eta}^{*} s_{\xi} \neq 0$ if and only if $\xi, \eta \in F_{\mu}$ for $\mu=\left(i_{0}, i_{1}, \ldots, i_{k}\right) \in$ $\Omega_{A}^{k}$ and $\xi-\eta=n A_{i_{k-1}, i_{k}} \xi_{\mu}^{k}$ for $n \in \mathbb{Z}$. In this case, we have $s_{\eta}^{*} s_{\xi}=u_{i_{k}}^{n}$.

Proof. Straightforward.

Let $\beta: \mathbb{T} \curvearrowright \mathcal{O}_{A, B}$ be the gauge action of $\mathcal{O}_{A, B}$ defined in Definition 4.1. We examine the structure of the fixed point algebra $\mathcal{O}_{A, B}^{\mathbb{T}}$ of the gauge action $\beta$. For $k \in \mathbb{N}$, we define $\mathcal{C}_{A, B}^{k} \subset \mathcal{O}_{A, B}$ by

$$
\mathcal{C}_{A, B}^{k}:=\overline{\operatorname{span}}\left\{s_{\xi} s_{\eta}^{*} \mid \xi, \eta \in \mathcal{F}^{k}\right\}
$$


By Lemma 5.1, $\mathcal{C}_{A, B}^{k}$ is a $C^{*}$-subalgebra of $\mathcal{O}_{A, B}$.

Lemma 5.2. For $k \in \mathbb{N}$, the $C^{*}$-algebra $\mathcal{C}_{A, B}^{k}$ is isomorphic to $\bigoplus_{i=1}^{N} C^{*}\left(u_{i}\right) \otimes \mathcal{K}\left(\ell^{2}\left(X_{i}^{k}\right)\right)$ where $\left\{X_{i}^{k}\right\}_{i=1}^{N}$ are subsets of $\mathcal{F}^{k}$ defined in the proof.

Proof. For $k \in \mathbb{N}$ and $i \in\{1,2, \ldots, N\}$, we define $\left(\Omega_{A}^{k}\right)_{i} \subset \Omega_{A}^{k}$ by

$$
\left(\Omega_{A}^{k}\right)_{i}:=\left\{\mu=\left(i_{0}, i_{1}, \ldots, i_{k}\right) \in \Omega_{A}^{k} \mid i_{k}=i\right\} .
$$

We define $\mathcal{F}_{i}^{k} \subset \mathcal{F}^{k}$ by $\mathcal{F}_{i}^{k}=\coprod_{\mu \in\left(\Omega_{A}^{k}\right)_{i}} F_{\mu}$. Note that we have $\mathcal{F}^{k}=\coprod_{i=1}^{N} \mathcal{F}_{i}^{k}$ and $\mathcal{F}_{i}^{k}=\left\{\xi \in \mathcal{F}^{k} \mid s_{\xi}^{*} s_{\xi}=p_{i}\right\}$ for each $i$. We define $\left(\mathcal{C}_{A, B}^{k}\right)_{i} \subset \mathcal{C}_{A, B}^{k}$ by

$$
\left(\mathcal{C}_{A, B}^{k}\right)_{i}:=\overline{\operatorname{span}}\left\{s_{\xi} s_{\eta}^{*} \mid \xi, \eta \in \mathcal{F}_{i}^{k}\right\} .
$$

Then we have $\mathcal{C}_{A, B}^{k} \cong \bigoplus_{i=1}^{N}\left(\mathcal{C}_{A, B}^{k}\right)_{i}$.

For $\mu \in \Omega_{A}^{0}$, we define $X_{\mu}:=\{0\} \subset F_{\mu}$. For $\mu=\left(i_{0}, i_{1}, \ldots, i_{k}\right) \in \Omega_{A}^{k}$ with a positive integer $k$, let $X_{\mu} \subset F_{\mu}$ be the image of the map

$$
\prod_{l=1}^{k}\left\{1,2, \ldots, A_{i_{l-1}, i_{l}}\right\} \ni\left(n_{l}\right)_{l=1}^{k} \mapsto \sum_{l=1}^{k} n_{l} \xi_{\mu}^{l} \in F_{\mu} .
$$

Then it is routine to see that the map above is injective, and each $\xi \in F_{\mu}$ can be uniquely written as $\xi=\eta+n A_{i_{k-1}, i_{k}} \xi_{\mu}^{k}$ for $\eta \in X_{\mu}$ and $n \in \mathbb{Z}$. Note that we have $s_{\xi}=s_{\eta} u_{i_{k}}^{n}$. For $k \in \mathbb{N}$ and $i \in\{1,2, \ldots, N\}$, we define a subset $X_{i}^{k} \subset \mathcal{F}_{i}^{k}$ by $X_{i}^{k}:=\coprod_{\mu \in\left(\Omega_{A}^{k}\right)_{i}} X_{\mu}$. Then for $\xi, \eta \in X_{i}^{k}$ with $\xi \neq \eta$ we have $s_{\eta}^{*} s_{\xi}=0$ by Lemma 5.1. Now it is routine to check that the map

$$
C^{*}\left(u_{i}\right) \otimes \mathcal{K}\left(\ell^{2}\left(X_{i}^{k}\right)\right) \ni x \otimes \theta_{\xi, \eta} \mapsto s_{\xi} x s_{\eta}^{*} \in\left(\mathcal{C}_{A, B}^{k}\right)_{i}
$$

is an isomorphism where $\left\{\theta_{\xi, \eta}\right\}_{\xi, \eta \in X_{i}^{k}}$ are the matrix units of $\mathcal{K}\left(\ell^{2}\left(X_{i}^{k}\right)\right)$. We are done.

We use the following lemma in the next section.

Lemma 5.3. For a positive element $a \in \mathcal{C}_{A, B}^{k}$ with $k \in \mathbb{N}$, there exists $b \in \overline{\operatorname{span}}\left\{s_{\xi} \mid \xi \in\right.$ $\left.\mathcal{F}^{k}\right\}$ such that $\|b\|=1,\left\|b^{*} a b\right\|=\|a\|$ and $b^{*} a b \in C^{*}\left(u_{i}\right)$ for some $i \in\{1,2, \ldots, N\}$.

Proof. We use the notation established in the proof of Lemma 5.2.

Take a positive element $a \in \mathcal{C}_{A, B}^{k}$ with $k \in \mathbb{N}$. There exist positive elements $a_{i} \in\left(\mathcal{C}_{A, B}^{k}\right)_{i}$ for $i \in\{1,2, \ldots, N\}$ such that $a=\sum_{i=1}^{N} a_{i}$ and $\|a\|=\max _{i}\left\|a_{i}\right\|$. Take $i$ with $\|a\|=\left\|a_{i}\right\|$. For each $z \in \mathbb{T}$ in the spectrum of $u_{i}$, we define a $*$-homomorphism $\varphi_{z}:\left(\mathcal{C}_{A, B}^{k}\right)_{i} \rightarrow$ $\mathcal{K}\left(\ell^{2}\left(X_{i}^{k}\right)\right)$ by $\varphi_{z}\left(s_{\xi} u_{i}^{n} s_{\eta}^{*}\right)=z^{n} \theta_{\xi, \eta}$ for $\xi, \eta \in X_{i}^{k}$ and $n \in \mathbb{Z}$. It is well-defined and we have $\|a\|=\left\|a_{i}\right\|=\max _{z}\left\|\varphi_{z}\left(a_{i}\right)\right\|$ by the proof of Lemma 5.2. Take $z \in \mathbb{T}$ with $\|a\|=\left\|\varphi_{z}\left(a_{i}\right)\right\|$. Since $\varphi_{z}\left(a_{i}\right) \in \mathcal{K}\left(\ell^{2}\left(X_{i}^{k}\right)\right)$ is positive, there exists $w \in \ell^{2}\left(X_{i}^{k}\right)$ such that $\|w\|=1$ and $\|a\|=\left\|\varphi_{z}\left(a_{i}\right)\right\|=\left\langle w, \varphi_{z}\left(a_{i}\right) w\right\rangle$ where $\langle\cdot, \cdot\rangle$ denotes the inner product in $\ell^{2}\left(X_{i}^{k}\right)$. Express $w \in \ell^{2}\left(X_{i}^{k}\right)$ as $w=\sum_{\xi \in X_{i}^{k}} \lambda_{\xi} \delta_{\xi}$ using $\lambda_{\xi} \in \mathbb{C}$ with $\sum_{\xi \in X_{i}^{k}}\left|\lambda_{\xi}\right|^{2}=1$. We set $b=\sum_{\xi \in X_{i}^{k}} \lambda_{\xi} s_{\xi} \in \mathcal{O}_{A, B}$ which converges in the norm topology and satisfies $\|b\|=1$. By Lemma 5.1, $b^{*} a b=b^{*} a_{i} b \in C^{*}\left(u_{i}\right)$. We obtain $\psi_{z}\left(b^{*} a_{i} b\right)=\left\langle w, \varphi_{z}\left(a_{i}\right) w\right\rangle=\|a\|$ where $\psi_{z}: C^{*}\left(u_{i}\right) \rightarrow \mathbb{C}$ is defined by $\psi_{z}\left(u_{i}\right)=z$. Hence we get $\left\|b^{*} a b\right\|=\|a\|$. Thus the element $b \in \mathcal{O}_{A, B}$ satisfies the desired conditions. 
Definition 5.4. We define a faithful conditional expectation $\Phi: \mathcal{O}_{A, B} \rightarrow \mathcal{O}_{A, B}^{\mathbb{T}}$ by

$$
\Phi(x):=\int_{\mathbb{T}} \beta_{z}(x) d z
$$

where $d z$ is the normalized Haar measure on $\mathbb{T}$.

For $\xi \in \mathcal{F}^{k}$, we get $\beta_{z}\left(s_{\xi}\right)=z^{k} s_{\xi}$. Hence for $\xi \in \mathcal{F}^{k}$ and $\eta \in \mathcal{F}^{l}$ we have

$$
\Phi\left(s_{\xi} s_{\eta}^{*}\right)= \begin{cases}s_{\xi} s_{\eta}^{*} & \text { if } k=l \\ 0 & \text { if } k \neq l\end{cases}
$$

Lemma 5.5. We have $\mathcal{C}_{A, B}^{k} \subset \mathcal{C}_{A, B}^{k+1}$ for each $k \in \mathbb{N}$, and $\mathcal{O}_{A, B}^{\mathbb{T}}=\overline{\bigcup_{k \in \mathbb{N}} \mathcal{C}_{A, B}^{k}}$.

Proof. By the relation (iii) in Definition 2.2, we have $\mathcal{C}_{A, B}^{k} \subset \mathcal{C}_{A, B}^{k+1}$. Since the conditional expectation $\Phi$ is bounded, $\Phi\left(\mathcal{O}_{A, B}^{\text {alg }}\right)$ is dense in $\mathcal{O}_{A, B}^{\mathbb{T}}$. By the computation of $\Phi\left(s_{\xi} s_{\eta}^{*}\right)$ above, we get $\Phi\left(\mathcal{O}_{A, B}^{\mathrm{alg}}\right) \subset \bigcup_{k \in \mathbb{N}} \mathcal{C}_{A, B}^{k}$. Thus we obtain $\mathcal{O}_{A, B}^{\mathbb{T}}=\overline{\bigcup_{k \in \mathbb{N}} \mathcal{C}_{A, B}^{k}}$.

Proposition 5.6. For $A, B \in M_{N}(\mathbb{Z})$ satisfying the condition (0), the $C^{*}$-algebra $\mathcal{O}_{A, B}$ is nuclear.

Proof. By Lemma 5.2 and Lemma 5.5, the $C^{*}$-algebra $\mathcal{O}_{A, B}^{\mathbb{T}}$ is nuclear. Hence $\mathcal{O}_{A, B}$ is nuclear by [DLRZ, Proposition 2].

\section{Pure infiniteness of $\mathcal{O}_{A, B}$}

In this section, we prove Proposition 2.10. We need the following lemma.

Lemma 6.1. Let $K$ be an integer greater than 1 . Let $\mathcal{A}$ be a unital $C^{*}$-algebra which has a unitary $u \in \mathcal{A}$ and an isometry $s \in \mathcal{A}$ satisfying that $s^{*} u^{n} s=0$ for $n=1,2, \ldots, K-1$ and $u^{K} s=$ su. Then for $k \in \mathbb{N}$ and a function $f \in C(\mathbb{T})$ which is 1 on some non-empty open subset of $\mathbb{T}$, there exist $k_{0} \in \mathbb{N}$ with $k_{0} \geq k$ and a function $g \in C(\mathbb{T})$ such that $v:=g(u) s^{k_{0}} \in \mathcal{A}$ is an isometry satisfying $f(u) v=v$ and $v^{*} u^{n} s^{l} v=0$ for all $n \in \mathbb{Z}$ and $l \in\{1,2, \ldots, k\}$.

Proof. We define a linear map $E: C(\mathbb{T}) \rightarrow C(\mathbb{T})$ by

$$
E(g)(z):=\frac{1}{K} \sum_{w \in \mathbb{T} \text { with } w^{K}=z} g(w)
$$

for $g \in C(\mathbb{T})$ and $z \in \mathbb{T}$. Then the map $E$ is characterized by the contracting linear map satisfying

$$
E\left(z^{n}\right)= \begin{cases}z^{m} & \text { if } n=K m \text { for } m \in \mathbb{Z} \\ 0 & \text { if } n \in \mathbb{Z} \backslash K \mathbb{Z},\end{cases}
$$

where $z \in C(\mathbb{T})$ is the identity function. Since we have $s^{*} u^{K m} s=u^{m}$ for $m \in \mathbb{Z}$ and $s^{*} u^{n} s=0$ for $n \in \mathbb{Z} \backslash K \mathbb{Z}$, we get $s^{*} g(u) s=E(g)(u)$ for all $g \in C(\mathbb{T})$.

Take $k \in \mathbb{N}$ and a function $f \in C(\mathbb{T})$ which is 1 on some non-empty open subset of $\mathbb{T}$. Then we can find a real number $t_{0}$, and $k_{0} \in \mathbb{N}$ with $k_{0} \geq k$ such that $f$ is 1 on

$$
I_{0}:=\left\{e^{2 \pi \sqrt{-1} t} \mid t_{0}-K^{-k_{0}} \leq t \leq t_{0}+K^{-k_{0}}\right\} \subset \mathbb{T},
$$

and $I_{0} \cap I_{l}=\emptyset$ for $l=1,2, \ldots, k$ where

$$
I_{l}:=\left\{e^{2 \pi \sqrt{-1} K^{l} t} \mid t_{0}-K^{-k_{0}} \leq t \leq t_{0}+K^{-k_{0}}\right\} \subset \mathbb{T} .
$$


Let us define a positive function $g_{0} \in C(\mathbb{T})$ by

$$
g_{0}(z):= \begin{cases}K^{k_{0}}-K^{2 k_{0}}\left|t-t_{0}\right| & \left(\text { for } z=e^{2 \pi \sqrt{-1} t} \text { with } t_{0}-K^{-k_{0}} \leq t \leq t_{0}+K^{-k_{0}}\right), \\ 0 & \left(\text { for } z \notin I_{0}\right) .\end{cases}
$$

Then for all $z \in \mathbb{T}$ we have

$$
E^{k_{0}}\left(g_{0}\right)(z)=\frac{1}{K^{k_{0}}} \sum_{w \in \mathbb{T} \text { with } w^{K^{k_{0}}}=z} g_{0}(w)=1 .
$$

Thus we obtain $E^{k_{0}}\left(g_{0}\right)=1$. We set $g:=g_{0}^{1 / 2} \in C(\mathbb{T})$. Then the element $v:=g(u) s^{k_{0}} \in \mathcal{A}$ satisfies $f(u) v=v$ because $f g=g$. From the computation $v^{*} v=\left(s^{*}\right)^{k_{0}} g_{0}(u) s^{k_{0}}=$ $E^{k_{0}}\left(g_{0}\right)(u)=1$, we see that $v$ is an isometry. We will show $g(u) s^{l} g(u)=0$ for $l \in$ $\{1,2, \ldots, k\}$. This implies $v^{*} u^{n} s^{l} v=0$ for all $n \in \mathbb{Z}$ and $l \in\{1,2, \ldots, k\}$, and hence completes the proof. Take $l \in\{1,2, \ldots, k\}$. We have $s^{l} g(u)=g\left(u^{K^{l}}\right) s^{l}=g_{l}(u) s^{l}$ where $g_{l} \in C(\mathbb{T})$ is defined by $g_{l}(z)=g\left(z^{K^{l}}\right)$ for $z \in \mathbb{T}$. If $z \in \mathbb{T}$ satisfies $g(z) \neq 0$ then we get $z \in I_{0}$. Hence $z^{K^{l}} \in I_{l}$. Since $I_{0} \cap I_{l}=\emptyset$, we have $z^{K^{l}} \notin I_{0}$. Therefore $g_{l}(z)=g\left(z^{K^{l}}\right)=0$. This shows $g g_{l}=0$. Thus we get $g(u) s^{l} g(u)=g(u) g_{l}(u) s^{l}=0$. We are done.

Proposition 6.2. Let $A, B \in M_{N}(\mathbb{Z})$ satisfy the conditions (0), (1) and (2) in Section 2. Then for every non-zero positive element $x \in \mathcal{O}_{A, B}$, there exist $a \in \mathcal{O}_{A, B}$ and $i \in\{1,2, \ldots, N\}$ with $a^{*} x a=p_{i}$.

Proof. Take a non-zero positive element $x \in \mathcal{O}_{A, B}$. To find $a \in \mathcal{O}_{A, B}$ and $i \in\{1,2, \ldots, N\}$ with $a^{*} x a=p_{i}$, it suffices to find $a_{0} \in \mathcal{O}_{A, B}, i \in\{1,2, \ldots, N\}$ and $C>0$ with $\| a_{0}^{*} x a_{0}-$ $C p_{i} \|<C$ because $p_{i}$ is a projection. Let $\varepsilon:=\|\Phi(x)\| / 3>0$ where $\Phi: \mathcal{O}_{A, B} \rightarrow \mathcal{O}_{A, B}^{\mathbb{T}}$ is the faithful conditional expectation defined in Definition 5.4. Choose a positive element $x_{0} \in \mathcal{O}_{A, B}^{\text {alg }}$ with $\left\|x-x_{0}\right\|<\varepsilon$. Set $C:=\left\|\Phi\left(x_{0}\right)\right\|$. We have

$$
C=\left\|\Phi\left(x_{0}\right)\right\|>\|\Phi(x)\|-\varepsilon=2 \varepsilon .
$$

Take $k \in \mathbb{N}$ with $x_{0} \in \operatorname{span}\left\{s_{\xi} s_{\eta}^{*} \mid \xi, \eta \in \coprod_{l=0}^{k} \mathcal{F}^{l}\right\}$. Then $\Phi\left(x_{0}\right) \in \mathcal{C}_{A, B}^{k}$. By Lemma 5.3, there exists $b \in \overline{\operatorname{span}}\left\{s_{\xi} \mid \xi \in \mathcal{F}^{k}\right\}$ such that $\|b\|=1,\left\|b^{*} \Phi\left(x_{0}\right) b\right\|=\left\|\Phi\left(x_{0}\right)\right\|=C$ and $b^{*} \Phi\left(x_{0}\right) b \in C^{*}\left(u_{i}\right)$ for some $i \in\{1,2, \ldots, N\}$. Take $f_{0} \in C(\mathbb{T})$ with $f_{0}\left(u_{i}\right)=b^{*} \Phi\left(x_{0}\right) b$ and $\left\|f_{0}\right\|=C$. Choose $f \in C(\mathbb{T})$ which is 1 on some non-empty open subset of $\mathbb{T}$, and satisfies $\left\|f_{0}-C f\right\|<\varepsilon$. Set $s:=s(0)_{i, i}$. Since $B_{i, i}=1$, two elements $u_{i}$ and $s$ satisfy the assumptions of Lemma 6.1 for $K=A_{i, i} \geq 2$ in the $C^{*}$-algebra generated by them. Applying Lemma 6.1 to $k \in \mathbb{N}$ and $f \in C(\mathbb{T})$, we get $k_{0} \in \mathbb{N}$ with $k_{0} \geq k$ and $g \in C(\mathbb{T})$ such that $v:=g\left(u_{i}\right) s^{k_{0}} \in \mathcal{O}_{A, B}$ satisfies $v^{*} v=p_{i}, f\left(u_{i}\right) v=v$ and $v^{*} u_{i}^{n} s^{l} v=0$ for all $n \in \mathbb{Z}$ and $l \in\{1,2, \ldots, k\}$. We set $a_{0}:=b v$. Then we have $\left\|a_{0}\right\| \leq 1$. We will show $a_{0}^{*} s_{\xi} s_{\eta}^{*} a_{0}=0$ for $\xi \in \mathcal{F}^{l_{1}}$ and $\eta \in \mathcal{F}^{l_{2}}$ with $l_{1}, l_{2} \leq k$ and $l_{1} \neq l_{2}$. We may assume $l_{1}>l_{2}$. We set $l:=l_{1}-l_{2} \in\{1,2, \ldots, k\}$. By Lemma 5.1 , we see

$$
b^{*} s_{\xi} s_{\eta}^{*} b \in \overline{\operatorname{span}}\left\{s_{\zeta} \mid \zeta \in \mathcal{F}^{l}\right\} .
$$

Hence it suffices to show $v^{*} s_{\zeta} v=0$ for all $\zeta \in \mathcal{F}^{l}$. Take $\mu \in \Omega_{A}^{l}$ with $\zeta \in F_{\mu}$. If $\mu \neq(i, i, \ldots, i)$ then $v^{*} s_{\zeta}=\left(s^{k_{0}}\right)^{*} g\left(u_{i}\right)^{*} s_{\zeta}=0$ by Lemma 5.1. If $\mu=(i, i, \ldots, i)$, then $s_{\zeta}=u_{i}^{n} s^{l}$ for some $n \in \mathbb{Z}$ because $B_{i, i}=1$. Hence we have $v^{*} s_{\zeta} v=0$. Thus we have shown $a_{0}^{*} s_{\xi} s_{\eta}^{*} a_{0}=0$ for $\xi \in \mathcal{F}^{l_{1}}$ and $\eta \in \mathcal{F}^{l_{2}}$ with $l_{1}, l_{2} \leq k$ and $l_{1} \neq l_{2}$. Therefore we obtain

$$
a_{0}^{*} x_{0} a_{0}=a_{0}^{*} \Phi\left(x_{0}\right) a_{0}=v^{*} f_{0}\left(u_{i}\right) v
$$


On the other hand, we have $v^{*} f\left(u_{i}\right) v=v^{*} v=p_{i}$. Thus we get $\left\|a_{0}^{*} x_{0} a_{0}-C p_{i}\right\|<\varepsilon$. because $\left\|f_{0}-C f\right\|<\varepsilon$. Hence

$$
\left\|a_{0}^{*} x a_{0}-C p_{i}\right\| \leq\left\|a_{0}^{*}\left(x-x_{0}\right) a_{0}\right\|+\left\|a_{0}^{*} x_{0} a_{0}-C p_{i}\right\|<2 \varepsilon<C .
$$

We are done.

Proof of Proposition 2.10. One can easily check that if $A, B$ satisfy the conditions (0), (1) and (2), the projection $p_{i} \in \mathcal{O}_{A, B}$ is full and properly infinite for all $i$. This fact and Proposition 6.2 show that the $C^{*}$-algebra $\mathcal{O}_{A, B}$ is simple and purely infinite.

\section{7. $K$-THEORY OF $\mathcal{O}_{A, B}$}

In this section, we give the proof of Proposition 2.6 using the idea in [Pi, Section 4]. Let us take $N \in \mathfrak{C}$ and $A, B \in M_{N}(\mathbb{Z})$ satisfying the condition (0).

Definition 7.1. We define a $C^{*}$-algebra $\mathcal{T}_{A, B}$ to be the universal $C^{*}$-algebra generated by mutually orthogonal projections $\left\{p_{i}\right\}_{i=1}^{N}$, partial unitaries $\left\{u_{i}\right\}_{i=1}^{N}$ with $u_{i}^{0}=p_{i}$, and partial isometries $\left\{s(n)_{i, j}\right\}_{(i, j) \in \Omega_{A}, n \in \mathbb{Z}}$ satisfying the relations (i), (ii) in Definition 2.2 and the relation

(iii)' $p_{i} \geq \sum_{j \in \Omega_{A}(i)} \sum_{n=1}^{A_{i, j}} s(n)_{i, j} s(n)_{i, j}^{*}$ for all $i$.

By the universality of $\mathcal{T}_{A, B}$, there exists a natural surjection $\mathcal{T}_{A, B} \rightarrow \mathcal{O}_{A, B}$ whose kernel is denoted by $\mathcal{J}_{A, B} \subset \mathcal{T}_{A, B}$. For each $i$, let us define $p_{i}^{\prime}, u_{i}^{\prime} \in \mathcal{T}_{A, B}$ by

$$
\begin{aligned}
& p_{i}^{\prime}:=p_{i}-\sum_{j \in \Omega_{A}(i)} \sum_{n=1}^{A_{i, j}} s(n)_{i, j} s(n)_{i, j}^{*}, \\
& u_{i}^{\prime}:=u_{i}-\sum_{j \in \Omega_{A}(i)} \sum_{n=1}^{A_{i, j}} s\left(n+B_{i, j}\right)_{i, j} s(n)_{i, j}^{*}=u_{i} p_{i}^{\prime}=p_{i}^{\prime} u_{i} .
\end{aligned}
$$

Then $p_{i}^{\prime}$ is a projection and $u_{i}^{\prime}$ is a partial unitary with $\left(u_{i}^{\prime}\right)^{0}=p_{i}^{\prime}$. Note that $p_{i}^{\prime}, u_{i}^{\prime} \in \mathcal{J}_{A, B}$ and $\mathcal{J}_{A, B}$ is generated by $\left\{p_{i}^{\prime}\right\}_{i=1}^{N}$ as an ideal.

Lemma 7.2. For $i \in\{1,2, \ldots, N\}$ and $\xi \in \mathcal{F}^{*}$, we have

$$
p_{i}^{\prime} s_{\xi}= \begin{cases}\left(u_{i}^{\prime}\right)^{n} & \text { if } \xi=n \xi_{(i)}^{0} \in F_{(i)} \text { for } n \in \mathbb{Z}, \\ 0 & \text { if } \xi \in F_{\mu} \text { with } \mu \in \Omega_{A}^{*} \backslash\{(i)\} .\end{cases}
$$

Proof. Straightforward.

Lemma 7.3. For each $i \in\{1,2, \ldots, N\}$, the spectrum of $u_{i}^{\prime} \in \mathcal{J}_{A, B} \subset \mathcal{T}_{A, B}$ contains $\mathbb{T}$.

Proof. The proof is very similar to the one of Proposition 4.2. It suffices to find operators $\left\{P_{i}\right\}_{i=1}^{N},\left\{U_{i}\right\}_{i=1}^{N}$, and $\left\{S(n)_{i, j}\right\}_{(i, j) \in \Omega_{A}, n \in \mathbb{Z}}$ on some Hilbert space such that they satisfy the relations (i), (ii) and (iii)' and the spectrum of

$$
U_{i}^{\prime}:=U_{i}-\sum_{j \in \Omega_{A}(i)} \sum_{n=1}^{A_{i, j}} S\left(n+B_{i, j}\right)_{i, j} S(n)_{i, j}^{*}
$$

contains $\mathbb{T}$ for each $i \in\{1,2, \ldots, N\}$.

For $(i, j) \in \Omega_{A}$ and $\mu=\left(i_{0}, i_{1}, \ldots, i_{k}\right) \in \Omega_{A}^{k}$ with $i_{0}=j$, we set $i \mu \in \Omega_{A}^{k+1}$ by $i \mu=\left(i, i_{0}, i_{1}, \ldots, i_{k}\right)$. For $\mu \in \Omega_{A}^{k}$ with a positive integer $k$, we define a homomorphism 
$\sigma_{i}: F_{\mu} \rightarrow F_{i \mu}$ by $\sigma_{i}\left(\xi_{\mu}^{l}\right)=\xi_{i \mu}^{l+1}$ for $l=1,2, \ldots, k$. We set $\xi_{\mu}^{0}:=B_{i_{0}, i_{1}} \xi_{\mu}^{1}$. Then we have $\sigma_{i}\left(\xi_{\mu}^{0}\right)=A_{i, j} \xi_{\mu}^{1}$. For $\mu=\left(i_{0}\right)=(j) \in \Omega_{A}^{0}$ and hence $i \mu=(i, j)$, we define a homomorphism $\sigma_{i}: F_{\mu} \rightarrow F_{i \mu}$ by $\sigma_{i}\left(\xi_{\mu}^{0}\right)=A_{i, j} \xi_{i \mu}^{1}$. Similarly as in the proof of Proposition 4.2 , we can see that $\sigma_{i}: F_{\mu} \rightarrow F_{i \mu}$ is injective, and $F_{i \mu}=\coprod_{n=1}^{A_{i, j}}\left(\sigma_{i}\left(F_{\mu}\right)+n \xi_{i \mu}^{1}\right)$.

We define operators $\left\{P_{i}\right\}_{i=1}^{N},\left\{U_{i}\right\}_{i=1}^{N}$, and $\left\{S(n)_{i, j}\right\}_{(i, j) \in \Omega_{A}, n \in \mathbb{Z}}$ on the Hilbert space $\ell^{2}\left(\mathcal{F}^{*}\right)$ by

$$
\begin{gathered}
P_{i}\left(\delta_{\xi}\right):=\left\{\begin{array}{ll}
\delta_{\xi} & \text { if } i_{0}=i \\
0 & \text { if } i_{0} \neq i,
\end{array} \quad U_{i}\left(\delta_{\xi}\right):= \begin{cases}\delta_{\xi+\xi_{\mu}^{0}} & \text { if } i_{0}=i \\
0 & \text { if } i_{0} \neq i,\end{cases} \right. \\
S(n)_{i, j}\left(\delta_{\xi}\right):= \begin{cases}\delta_{\sigma_{i}(\xi)+n \xi_{i \mu}^{1}} & \text { if } i_{0}=j \\
0 & \text { if } i_{0} \neq j,\end{cases}
\end{gathered}
$$

for $\xi \in F_{\mu} \subset \mathcal{F}^{*}$ with $\mu=\left(i_{0}, i_{1}, \ldots, i_{k}\right) \in \Omega_{A}^{k}$. Then it is routine to check that these operators satisfy the relations (i), (ii) and (iii)'. We see that for each $i \in\{1,2, \ldots, N\}$ the operator $U_{i}^{\prime} \in B\left(\ell^{2}\left(\mathcal{F}^{*}\right)\right)$ acts as a bilateral shift on $\ell^{2}\left(F_{(i)}\right) \cong \ell^{2}(\mathbb{Z})$ and vanishes on $\ell^{2}\left(\mathcal{F}^{*} \backslash F_{(i)}\right)$. Hence the spectrum of $U_{i}^{\prime} \in B\left(\ell^{2}\left(\mathcal{F}^{*}\right)\right)$ contains $\mathbb{T}$. We are done.

Remark 7.4. By Corollary A.2, we see that the representation of $\mathcal{T}_{A, B}$ defined from the operators in the proof above is faithful. We use this fact in Appendix A.

Definition 7.5. For $N \in \mathfrak{C}$, we define a $C^{*}$-algebra $\mathcal{A}_{N}$ to be the universal $C^{*}$-algebra generated by mutually orthogonal projections $\left\{p_{i}\right\}_{i=1}^{N}$ and partial unitaries $\left\{u_{i}\right\}_{i=1}^{N}$ with $u_{i}^{0}=p_{i}$.

Clearly we have $\mathcal{A}_{N} \cong C_{0}(\{1,2, \ldots, N\} \times \mathbb{T})$. By definition, there exists a $*$-homomorphism $\mathcal{A}_{N} \rightarrow \mathcal{T}_{A, B}$ which sends $\left\{p_{i}, u_{i}\right\}_{i=1}^{N}$ to $\left\{p_{i}, u_{i}\right\}_{i=1}^{N}$. By Lemma 7.3 , this $*$-homomorphism is injective. Hence we can consider $\mathcal{A}_{N}$ as a $C^{*}$-subalgebra of $\mathcal{T}_{A, B}$. Note that the natural *-homomorphism $\mathcal{A}_{N} \rightarrow \mathcal{O}_{A, B}$ is injective only when $B$ has no zero rows by Corollary 4.3 .

Lemma 7.6. The inclusion $\mathcal{A}_{N} \hookrightarrow \mathcal{T}_{A, B}$ is a $K K$-equivalence.

Proof. See Appendix A.

Lemma 7.7. The $*$-homomorphism $\pi: \mathcal{A}_{N} \rightarrow \mathcal{J}_{A, B}$ defined by $\pi\left(p_{i}\right)=p_{i}^{\prime}$ and $\pi\left(u_{i}\right)=u_{i}^{\prime}$ for $i=1,2, \ldots, N$ is an injection onto a hereditary and full $C^{*}$-subalgebra.

Proof. By Lemma 7.3, the $*$-homomorphism $\pi$ is an injection onto the $C^{*}$-subalgebra of $\mathcal{J}_{A, B}$ generated by $\left\{p_{i}^{\prime}, u_{i}^{\prime}\right\}_{i=1}^{N}$. This $C^{*}$-subalgebra is the hereditary $C^{*}$-subalgebra of $\mathcal{T}_{A, B}$ generated by $\left\{p_{i}^{\prime}\right\}_{i=1}^{N}$ by Lemma 7.2. Thus it is hereditary and full in $\mathcal{J}_{A, B}$ because $\mathcal{J}_{A, B}$ is generated by $\left\{p_{i}^{\prime}\right\}_{i=1}^{N}$ as an ideal.

Proposition 7.8. The $C^{*}$-algebra $\mathcal{O}_{A, B}$ is in the UCT class.

Proof. Since the $C^{*}$-algebra $\mathcal{A}_{N}$ is commutative, Lemma 7.6 and Lemma 7.7 show that $\mathcal{T}_{A, B}$ and $\mathcal{J}_{A, B}$ are in the UCT class (see [R $\varnothing$, Definition 2.4.5]). Now the "two out of three principle" ([Rø, Proposition 2.4.7 (i)]) shows that $\mathcal{O}_{A, B} \cong \mathcal{T}_{A, B} / \mathcal{J}_{A, B}$ is in the UCT class.

Lemma 7.9. The homomorphisms $K_{0}\left(\mathcal{J}_{A, B}\right) \rightarrow K_{0}\left(\mathcal{T}_{A, B}\right)$ and $K_{1}\left(\mathcal{J}_{A, B}\right) \rightarrow K_{1}\left(\mathcal{T}_{A, B}\right)$ induced by the embedding $\mathcal{J}_{A, B} \hookrightarrow \mathcal{T}_{A, B}$ coincide with $I-A: \mathbb{Z}^{N} \rightarrow \mathbb{Z}^{N}$ and $I-B: \mathbb{Z}^{N} \rightarrow$ $\mathbb{Z}^{N}$, respectively. 
Proof. By Lemma 7.6 and Lemma 7.7, all of the four abelian groups $K_{0}\left(\mathcal{T}_{A, B}\right), K_{1}\left(\mathcal{T}_{A, B}\right)$, $K_{0}\left(\mathcal{J}_{A, B}\right)$ and $K_{1}\left(\mathcal{J}_{A, B}\right)$ are isomorphic to $\mathbb{Z}^{N}$ whose basis are given by $\left\{\left[p_{i}\right]\right\}_{i=1}^{N},\left\{\left[u_{i}\right]\right\}_{i=1}^{N}$, $\left\{\left[p_{i}^{\prime}\right]\right\}_{i=1}^{N}$ and $\left\{\left[u_{i}^{\prime}\right]\right\}_{i=1}^{N}$, respectively.

For each $i$, we have

$$
\left[p_{i}^{\prime}\right]=\left[p_{i}\right]-\sum_{j \in \Omega_{A}(i)} \sum_{n=1}^{A_{i, j}}\left[s(n)_{i, j} s(n)_{i, j}^{*}\right]=\left[p_{i}\right]-\sum_{j=1}^{N} A_{i, j}\left[p_{j}\right] .
$$

This shows the result on $K_{0}$. For $(i, j) \in \Omega_{A}$, we define a partial unitary

$$
u_{i, j}:=\sum_{n=1}^{A_{i, j}} s(n+1)_{i, j} s(n)_{i, j}^{*} \in \mathcal{O}_{A, B} .
$$

Then we have $\left[u_{i, j}\right]=\left[u_{j}\right]$ (see Lemma B.2). Hence for each $i$, we get

$$
\left[u_{i}^{\prime}\right]=\left[u_{i}\right]-\sum_{j \in \Omega_{A}(i)}\left[u_{i, j}^{B_{i, j}}\right]=\left[u_{i}\right]-\sum_{j=1}^{N} B_{i, j}\left[u_{j}\right]
$$

(see Subsection 1.3). Thus we get the conclusion for $K_{1}$.

Proposition 7.10. We have $\Gamma_{A, B}$-equivariant exact sequences

$$
\begin{aligned}
& 0 \longrightarrow \operatorname{coker}(I-A) \longrightarrow K_{0}\left(\mathcal{O}_{A, B}\right) \longrightarrow \operatorname{ker}(I-B) \longrightarrow 0, \\
& 0 \longrightarrow \operatorname{coker}(I-B) \longrightarrow K_{1}\left(\mathcal{O}_{A, B}\right) \longrightarrow \operatorname{ker}(I-A) \longrightarrow 0
\end{aligned}
$$

both of which have $\Gamma_{A, B}$-equivariant splitting maps.

Proof. The same formula as $\Gamma_{A, B} \curvearrowright \mathcal{O}_{A, B}$ defines an action $\Gamma_{A, B} \curvearrowright \mathcal{T}_{A, B}$ which makes the short exact sequence

$$
0 \longrightarrow \mathcal{J}_{A, B} \longrightarrow \mathcal{T}_{A, B} \longrightarrow \mathcal{O}_{A, B} \longrightarrow 0
$$

$\Gamma_{A, B}$-equivariant. From this sequence, we get a $\Gamma_{A, B}$-equivariant 6 -term exact sequence

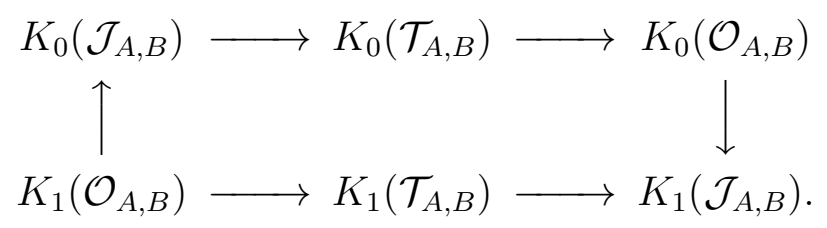

By Lemma 7.9 and its proof, the homomorphisms $K_{0}\left(\mathcal{J}_{A, B}\right) \rightarrow K_{0}\left(\mathcal{T}_{A, B}\right)$ and $K_{0}\left(\mathcal{J}_{A, B}\right) \rightarrow$ $K_{0}\left(\mathcal{T}_{A, B}\right)$ coincide with $I-A: \mathbb{Z}^{N} \rightarrow \mathbb{Z}^{N}$ and $I-B: \mathbb{Z}^{N} \rightarrow \mathbb{Z}^{N}, \Gamma_{A, B}$-equivariantly. Thus we get the desired $\Gamma_{A, B}$-equivariant short exact sequences. Since $\operatorname{ker}(I-A)$ and $\operatorname{ker}(I-B)$ are free abelian groups, these sequences split. That we can find $\Gamma_{A, B}$-equivariant splitting maps will be proven in Appendix B.

Remark 7.11. In the proof of main theorem in Section 3, we only use Proposition 7.10 for the case $\operatorname{ker}(I-A)=\operatorname{ker}(I-B)=0$ and hence we do not need the results on $\Gamma_{A, B^{-}}$ equivariant splitting maps. However in order to compute examples, it is important to show that there exist $\Gamma_{A, B}$-equivariant splitting maps. 


\section{Appendix A. Proof of Lemma 7.6}

In this appendix, we give a proof of Lemma 7.6 in an explicit way, following the argument in [Ka3, Appendix C] which was inspired by the original proof in [Pi]. We first need the following proposition which has own importance.

Proposition A.1. For $A, B \in M_{N}(\mathbb{Z})$ satisfying the condition (0), the ideal $\mathcal{J}_{A, B}$ of $\mathcal{T}_{A, B}$ is essential.

Proof. We use the notation established in the proof of Lemma 5.2.

Let $\mathcal{J}_{A, B}^{\perp}$ be the ideal of $\mathcal{T}_{A, B}$ defined by

$$
\mathcal{J}_{A, B}^{\perp}:=\left\{x \in \mathcal{T}_{A, B} \mid x y=0 \text { for all } y \in \mathcal{J}_{A, B}\right\} .
$$

We will show $\mathcal{J}_{A, B}^{\perp}=0$. The same formula of the gauge action $\beta: \mathbb{T} \curvearrowright \mathcal{O}_{A, B}$ defined in Definition 4.1 defines an action $\mathbb{T} \curvearrowright \mathcal{T}_{A, B}$. Since $\mathcal{J}_{A, B}$ is invariant under this action, so is $\mathcal{J}_{A, B}^{\perp}$. Hence in order to show $\mathcal{J}_{A, B}^{\perp}=0$, it suffices to see $\mathcal{J}_{A, B}^{\perp} \cap \mathcal{T}_{A, B}^{\mathbb{T}}=0$ where $\mathcal{T}_{A, B}^{\mathbb{T}}$ is the fixed point algebra of the action $\mathbb{T} \curvearrowright \mathcal{T}_{A, B}$.

For each $k \in \mathbb{N}$, we define $C^{*}$-subalgebras $\mathcal{C}_{A, B}^{k}, \mathcal{D}_{A, B}^{k}$ of $\mathcal{T}_{A, B}$ by

$$
\begin{aligned}
\mathcal{C}_{A, B}^{k} & :=\overline{\operatorname{span}}\left\{s_{\xi} s_{\eta}^{*} \mid \xi, \eta \in \mathcal{F}^{k}\right\} \\
& =\overline{\operatorname{span}}\left\{s_{\xi} p_{i} s_{\eta}^{*} \mid \xi, \eta \in \mathcal{F}_{i}^{k}, i \in\{1,2, \ldots, N\}\right\}, \\
\mathcal{D}_{A, B}^{k} & :=\overline{\operatorname{span}}\left\{s_{\xi} p_{i}^{\prime} s_{\eta}^{*} \mid \xi, \eta \in \mathcal{F}_{i}^{k}, i \in\{1,2, \ldots, N\}\right\} .
\end{aligned}
$$

Similarly as in Lemma 5.5, we have

$$
\mathcal{T}_{A, B}^{\mathbb{T}}=\overline{\bigcup_{k \in \mathbb{N}}\left(\sum_{l=0}^{k} \mathcal{C}_{A, B}^{l}\right)}
$$

Hence in order to show $\mathcal{J}_{A, B}^{\perp} \cap \mathcal{T}_{A, B}^{\mathbb{T}}=0$, it suffices to see $\mathcal{J}_{\bar{A}, B}^{\perp} \cap\left(\sum_{l=0}^{k} \mathcal{C}_{A, B}^{l}\right)=0$ for all $k \in \mathbb{N}$. This reduces to the problem showing $\mathcal{J}_{A, B}^{\perp} \cap \mathcal{C}_{A, B}^{k}=0$ because the $C^{*}$-algebra $\sum_{l=0}^{k} \mathcal{C}_{A, B}^{l}$ has the orthogonal decomposition

$$
\sum_{l=0}^{k} \mathcal{C}_{A, B}^{l}=\sum_{l=0}^{k-1} \mathcal{D}_{A, B}^{l}+\mathcal{C}_{A, B}^{k}
$$

and $\mathcal{D}_{A, B}^{l} \subset \mathcal{J}_{A, B}$ for all $l$.

Fix $k \in \mathbb{N}$, and we will show $\mathcal{J}_{A, B}^{\perp} \cap \mathcal{C}_{A, B}^{k}=0$. Similarly as in the proof of Lemma 5.2, we obtain

$$
\mathcal{C}_{A, B}^{k} \cong \bigoplus_{i=1}^{N} C^{*}\left(u_{i}\right) \otimes \mathcal{K}\left(\ell^{2}\left(X_{i}^{k}\right)\right), \quad \mathcal{D}_{A, B}^{k} \cong \bigoplus_{i=1}^{N} C^{*}\left(u_{i}^{\prime}\right) \otimes \mathcal{K}\left(\ell^{2}\left(X_{i}^{k}\right)\right) .
$$

By Lemma $7.3, C^{*}\left(u_{i}\right) \cong C^{*}\left(u_{i}^{\prime}\right) \cong C(\mathbb{T})$ for each $i$. This observation and the proof of Lemma 5.2 imply that the $*$-homomorphism $\varphi: \mathcal{C}_{A, B}^{k} \rightarrow \mathcal{D}_{A, B}^{k}$ defined by

$$
\varphi\left(s_{\xi} p_{i} s_{\eta}^{*}\right):=s_{\xi} p_{i}^{\prime} s_{\eta}^{*}
$$


for $\xi, \eta \in \mathcal{F}_{i}^{k}$ and $i \in\{1,2, \ldots, N\}$ is an isomorphism. For each $i \in\{1,2, \ldots, N\}$, we define a projection $p_{i}^{\prime \prime} \in \mathcal{T}_{A, B}$ by

$$
p_{i}^{\prime \prime}:=p_{i}-\sum_{\mu \in \Omega_{A}^{k+1}(i)} \sum_{\xi \in X_{\mu}} s_{\xi} s_{\xi}^{*}
$$

where $\Omega_{A}^{k+1}(i)$ is defined by

$$
\Omega_{A}^{k+1}(i):=\left\{\mu=\left(i_{0}, i_{1}, \ldots, i_{k+1}\right) \in \Omega_{A}^{k+1} \mid i_{0}=i\right\}
$$

which is a finite set by the condition (0). Then we have $p_{i_{0}}^{\prime \prime} s_{\xi}=s_{\xi} p_{i_{k}}^{\prime}$ for $\xi \in F_{\mu}$ with $\mu=\left(i_{0}, i_{1}, \ldots, i_{k}\right) \in \mathcal{F}^{k}$. This implies that the isomorphism $\varphi$ satisfies

$$
\varphi(x)=\sum_{i=1}^{N} p_{i}^{\prime \prime} x=\sum_{i=1}^{N} x p_{i}^{\prime \prime}
$$

for $x \in \mathcal{C}_{A, B}^{k}$. Since $p_{i}^{\prime \prime} \in \mathcal{J}_{A, B}$ for all $i, \mathcal{C}_{A, B}^{k} \cap \mathcal{J}_{A, B}^{\perp}$ is contained in the kernel of $\varphi$. Thus we have $\mathcal{C}_{A, B}^{k} \cap \mathcal{J}_{A, B}^{\perp}=0$. This completes the proof.

Corollary A.2. A*-homomorphism $\varphi$ from the $C^{*}$-algebra $\mathcal{T}_{A, B}$ is injective if and only if the spectrum of $\varphi\left(u_{i}^{\prime}\right)$ contains $\mathbb{T}$ for every $i \in\{1,2, \ldots, N\}$.

Proof. By Proposition A.1, $\varphi$ is injective if and only if its restriction to $\mathcal{J}_{A, B}$ is injective. This happens exactly when the restriction of $\varphi$ to the image of the $*$-homomorphism $\pi: \mathcal{A}_{N} \rightarrow \mathcal{J}_{A, B}$ in Lemma 7.7 is injective. This condition is equivalent that the spectrum of $\varphi\left(u_{i}^{\prime}\right)$ contains $\mathbb{T}$ for every $i \in\{1,2, \ldots, N\}$.

Recall that for two (separable) $C^{*}$-algebras $\mathcal{A}$ and $\mathcal{B}$, the $K K$-group $K K(\mathcal{A}, \mathcal{B})$ is an abelian group such that a homotopy equivalence class of a $*$-homomorphism $\varphi: \mathcal{A} \rightarrow \mathcal{B}$ defines an element $[\varphi]$ of $K K(\mathcal{A}, \mathcal{B})$ (see [Bl, Chapter VIII] for definitions and results stated below). We say that a $*$-homomorphism $\varphi: \mathcal{A} \rightarrow \mathcal{B}$ is a $K K$-equivalence if the element $[\varphi] \in K K(\mathcal{A}, \mathcal{B})$ has an inverse in $K K(\mathcal{B}, \mathcal{A})$ with respect to the Kasparov product. The facts on $K K$-equivalences we use in the following argument are summarized as follows:

- A composition of two $K K$-equivalences is a $K K$-equivalence.

- Let $\varphi_{i}: \mathcal{A}_{i} \rightarrow \mathcal{A}_{i+1}$ for $i=1,2,3$ be $*$-homomorphisms. If both $\varphi_{2} \circ \varphi_{1}$ and $\varphi_{3} \circ \varphi_{2}$ are $K K$-equivalences, then all the three $*$-homomorphisms $\varphi_{1}, \varphi_{2}, \varphi_{3}$ are $K K$-equivalences.

- An inclusion map of a hereditary and full $C^{*}$-subalgebra is a $K K$-equivalence.

- Let $0 \rightarrow \mathcal{I} \rightarrow \mathcal{A} \rightarrow \mathcal{B} \rightarrow 0$ be a splitting short exact sequence. If $\mathcal{B}$ is contractible, then the map $\mathcal{I} \rightarrow \mathcal{A}$ is a $K K$-equivalence.

- A *-homomorphism $\mathcal{A} \rightarrow \mathcal{B}$ is a $K K$-equivalence if and only if the induced $*$-homomorphism $S \mathcal{A} \rightarrow S \mathcal{B}$ is a $K K$-equivalence.

Here we define $S \mathcal{A}=C_{0}((0,1), \mathcal{A})$ for a $C^{*}$-algebra $\mathcal{A}$. A $K K$-equivalence induces isomorphisms of $K$-groups, and conversely a $*$-homomorphism $\mathcal{A} \rightarrow \mathcal{B}$ inducing isomorphisms of $K$-groups is a $K K$-equivalence if $\mathcal{A}$ and $\mathcal{B}$ are in the UCT class. It is easy to see that the five statements above still valid if we replace " $K K$-equivalence" to "*-homomorphism inducing isomorphisms of $K$-groups". 
Let us take $A, B \in M_{N}(\mathbb{Z})$ satisfying the condition (0). We will show that the inclusion $\mathcal{A}_{N} \hookrightarrow \mathcal{T}_{A, B}$ is a $K K$-equivalence. For $k, l \in \mathbb{N}$, we define

$$
\Omega_{A}^{k, l}:=\left\{\left(\mu, \mu^{\prime}\right) \mid \mu=\left(i_{0}, i_{1}, \ldots, i_{k}\right) \in \Omega_{A}^{k}, \mu^{\prime}=\left(i_{0}^{\prime}, i_{1}^{\prime}, \ldots, i_{l}^{\prime}\right) \in \Omega_{A}^{l} \text { with } i_{k}=i_{0}^{\prime}\right\} .
$$

Note that the map

$$
\Omega_{A}^{k, l} \ni\left(\mu, \mu^{\prime}\right) \mapsto \mu \mu^{\prime} \in \Omega_{A}^{k+l}
$$

is bijective where $\mu \mu^{\prime}=\left(i_{0}, i_{1}, \ldots, i_{k}, i_{1}^{\prime}, i_{2}^{\prime}, \ldots, i_{l}^{\prime}\right)$ for $\mu=\left(i_{0}, i_{1}, \ldots, i_{k}\right) \in \Omega_{A}^{k}$ and $\mu^{\prime}=$ $\left(i_{0}^{\prime}, i_{1}^{\prime}, \ldots, i_{l}^{\prime}\right) \in \Omega_{A}^{l}$. For $\left(\mu, \mu^{\prime}\right) \in \Omega_{A}^{k, l}$, we set $F_{\mu, \mu^{\prime}}:=F_{\mu \mu^{\prime}}$. Note that there exists a homomorphism $F_{\mu} \rightarrow F_{\mu, \mu^{\prime}}$ which sends $\xi_{\mu}^{k}$ to $\xi_{\mu \mu^{\prime}}^{k}$. We set $\Omega_{A}^{*, *}:=\coprod_{k, l \in \mathbb{N}} \Omega_{A}^{k, l}$ and $\mathcal{F}^{*, *}:=\coprod_{\left(\mu, \mu^{\prime}\right) \in \Omega_{A}^{* * *}} F_{\mu, \mu^{\prime}}$.

Take $(i, j) \in \Omega_{A}$ and $\left(\mu, \mu^{\prime}\right) \in \Omega_{A}^{k, l}$ with $i_{0}=j$ where $\mu=\left(i_{0}, i_{1}, \ldots, i_{k}\right)$. Note that the element $i \mu \in \Omega_{A}^{k+1}$ defined in the proof of Lemma 7.3 is nothing but $(i, j) \mu$ defined here, and that we have $(i \mu) \mu^{\prime}=i\left(\mu \mu^{\prime}\right)$. We denote by the same symbol $\sigma_{i}$ the homomorphism $F_{\mu, \mu^{\prime}} \rightarrow F_{i \mu, \mu^{\prime}}$ induced by the homomorphism $\sigma_{i}: F_{\mu \mu^{\prime}} \rightarrow F_{i \mu \mu^{\prime}}$ defined in the proof of Lemma 7.3. We define a $*$-homomorphism $\rho: \mathcal{T}_{A, B} \rightarrow B\left(\ell^{2}\left(\mathcal{F}^{*, *}\right)\right)$ by

$$
\begin{aligned}
\rho\left(p_{i}\right)\left(\delta_{\xi}\right) & :=\left\{\begin{array}{ll}
\delta_{\xi} & \text { if } i_{0}=i \\
0 & \text { if } i_{0} \neq i,
\end{array} \quad \rho\left(u_{i}\right)\left(\delta_{\xi}\right):= \begin{cases}\delta_{\xi+\xi_{\mu \mu^{\prime}}^{0}} & \text { if } i_{0}=i \\
0 & \text { if } i_{0} \neq i,\end{cases} \right. \\
\rho\left(s(n)_{i, j}\right)\left(\delta_{\xi}\right) & := \begin{cases}\delta_{\sigma_{i}(\xi)+n \xi_{i \mu \mu^{\prime}}^{1}} & \text { if } i_{0}=j \\
0 & \text { if } i_{0} \neq j,\end{cases}
\end{aligned}
$$

for generators $\left\{p_{i}, u_{i}, s(n)_{i, j}\right\}$ of $\mathcal{T}_{A, B}$ and $\xi \in F_{\mu, \mu^{\prime}} \subset \mathcal{F}^{*, *}$ with $\mu=\left(i_{0}, i_{1}, \ldots, i_{k}\right)$. It is routine to check that this is well-defined.

Since we can naturally identify $\Omega_{A}^{0, l}$ and $\Omega_{A}^{l}$, we consider $\Omega_{A}^{*}$ as a subset of $\Omega_{A}^{*, *}$ by $\Omega_{A}^{*}=\coprod_{l \in \mathbb{N}} \Omega_{A}^{0, l} \subset \Omega_{A}^{*, *}$. Using this identification, we can consider $\mathcal{F}^{*}$ as a subset of $\mathcal{F}^{*, *}$. Then the representation defined from the operators in the proof of Lemma 7.3 induces the representation $\mathcal{T}_{A, B} \rightarrow B\left(\ell^{2}\left(\mathcal{F}^{*}\right)\right) \subset B\left(\ell^{2}\left(\mathcal{F}^{*, *}\right)\right)$ which is denoted by $\tilde{\pi}$. By Corollary A.2, the representation $\tilde{\pi}$ is faithful. It is routine to check $\widetilde{\pi}\left(p_{i}\right)=\rho\left(p_{i}^{\prime}\right)$ and $\tilde{\pi}\left(u_{i}\right)=\rho\left(u_{i}^{\prime}\right)$ for $i \in\{1,2, \ldots, N\}$. Let $\mathcal{L} \subset B\left(\ell^{2}\left(\mathcal{F}^{*, *}\right)\right)$ be the $C^{*}$-algebra generated by $\tilde{\pi}\left(\mathcal{T}_{A, B}\right) \cup \rho\left(\mathcal{T}_{A, B}\right)$. For $\xi \in \mathcal{F}^{k}$ with $k \geq 1$ and $i \in\{1,2, \ldots, N\}$, we have $\tilde{\pi}\left(p_{i}\right) \rho\left(s_{\xi}\right)=\rho\left(p_{i}^{\prime} s_{\xi}\right)=0$ by Lemma 7.2. This fact shows that $\widetilde{\pi}\left(\mathcal{T}_{A, B}\right) \subset \mathcal{L}$ is the hereditary $C^{*}$-subalgebra of $\mathcal{L}$ generated by $\left\{\tilde{\pi}\left(p_{i}\right)\right\}_{i=1}^{N}$. We let $\mathcal{K} \subset \mathcal{L}$ be the ideal of $\mathcal{L}$ generated by $\left\{\tilde{\pi}\left(p_{i}\right)\right\}_{i=1}^{N}$. We have $\rho\left(\mathcal{J}_{A, B}\right) \subset \mathcal{K}$ because $\rho\left(p_{i}^{\prime}\right)=\tilde{\pi}\left(p_{i}\right)$ for all $i$.

Let $\pi: \mathcal{A}_{N} \rightarrow \mathcal{J}_{A, B}$ be the $*$-homomorphism defined in Lemma 7.7. Then the restriction of $\tilde{\pi}$ to $\mathcal{A}_{N} \subset \mathcal{T}_{A, B}$ coincides with $\rho \circ \pi$. Thus we get the following commutative diagram:

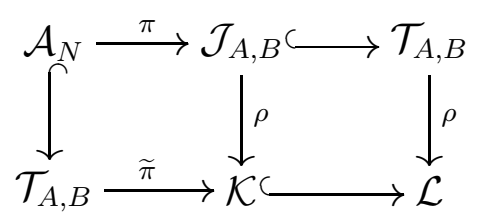

We define

$$
\begin{aligned}
& \mathcal{D}:=\left\{f \in C_{0}\left((-1,1), \mathcal{T}_{A, B}\right) \mid f(s)-f(-s) \in \mathcal{J}_{A, B} \text { for all } s \in(-1,1)\right\}, \\
& \widetilde{\mathcal{D}}:=\left\{f \in C_{0}((-1,1), \mathcal{L}) \mid f(s)-f(-s) \in \mathcal{K} \text { for all } s \in(-1,1)\right\} .
\end{aligned}
$$


Then $\rho$ induces the $*$-homomorphism $\mathcal{D} \rightarrow \widetilde{\mathcal{D}}$ which is also denoted by $\rho$. We have the following splitting short exact sequences;

$$
\begin{aligned}
& 0 \longrightarrow S \mathcal{J}_{A, B} \longrightarrow \mathcal{D} \longrightarrow C_{0}\left((-1,0], \mathcal{T}_{A, B}\right) \longrightarrow 0, \\
& 0 \longrightarrow C_{0}((-1,0], \mathcal{L}) \longrightarrow 0 .
\end{aligned}
$$

Since $C_{0}\left((-1,0], \mathcal{T}_{A, B}\right)$ is contractible, the inclusion map $S \mathcal{J}_{A, B} \hookrightarrow \mathcal{D}$ is a $K K$-equivalence. The *-homomorphism $S \mathcal{A}_{N} \rightarrow S \mathcal{J}_{A, B}$ induced by the $*$-homomorphism $\pi$ is a $K K$ equivalence because so is $\pi$ by Lemma 7.7. Hence the composition of these two $*$-homomorphisms which is denoted by $\varphi: S \mathcal{A}_{N} \rightarrow \mathcal{D}$ is a $K K$-equivalence. Since the map $\tilde{\pi}: \mathcal{T}_{A, B} \rightarrow \mathcal{K}$ is an injection onto a full and hereditary $C^{*}$-subalgebra of $\mathcal{K}$, we can show in a very similar way as above that the $*$-homomorphism $\widetilde{\varphi}: S \mathcal{T}_{A, B} \rightarrow \widetilde{\mathcal{D}}$ induced by $\tilde{\pi}$ is a $K K$-equivalence. We get $\tilde{\varphi} \circ \iota=\rho \circ \varphi$ where $\iota: S \mathcal{A}_{N} \hookrightarrow S \mathcal{T}_{A, B}$ is the embedding map.

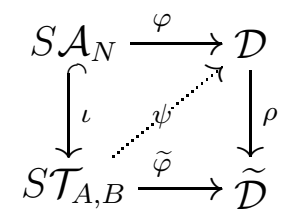

We will construct a $*$-homomorphism $\psi: S \mathcal{T}_{A, B} \rightarrow \mathcal{D}$ and show that $\psi \circ \iota$ and $\rho \circ \psi$ induces the same elements as $\varphi$ and $\widetilde{\varphi}$ in $K K$-groups, respectively. From this fact, we see that the map $\iota: S \mathcal{A}_{N} \hookrightarrow S \mathcal{T}_{A, B}$, and hence the inclusion $\mathcal{A}_{N} \hookrightarrow \mathcal{T}_{A, B}$ is a $K K$-equivalence.

We define an endomorphism $\theta: \mathcal{T}_{A, B} \rightarrow \mathcal{T}_{A, B}$ by

$$
\begin{aligned}
\theta\left(p_{i}\right) & :=\sum_{j \in \Omega_{A}(i)} \sum_{n=1}^{A_{i, j}} s(n)_{i, j} s(n)_{i, j}^{*}=p_{i}-p_{i}^{\prime} \\
\theta\left(u_{i}\right) & :=\sum_{n=1}^{A_{i, j}} s\left(n+B_{i, j}\right)_{i, j} s(n)_{i, j}^{*}=u_{i}-u_{i}^{\prime}=u_{i} \theta\left(p_{i}\right) \\
\theta\left(s(n)_{i, j}\right) & :=s(n)_{i, j} \theta\left(p_{j}\right)
\end{aligned}
$$

for generators $\left\{p_{i}, u_{i}, s(n)_{i, j}\right\}$ of $\mathcal{T}_{A, B}$. We can show that it is well-defined and satisfies $x-\theta(x) \in \mathcal{J}_{A, B}$ for all $x \in \mathcal{T}_{A, B}$ because this holds for the generators. Hence we can define a $*$-homomorphism $\psi: S \mathcal{T}_{A, B} \rightarrow \mathcal{D}$ by

$$
\psi(f)(s)= \begin{cases}f(s) & \text { for } s \in[0,1), \\ \theta(f(-s)) & \text { for } s \in(-1,0]\end{cases}
$$

for $f \in S \mathcal{T}_{A, B}$. The proof of Lemma 7.6 completes once we see that $\psi \circ \iota$ and $\rho \circ \psi$ induce the same elements in $K K$-groups as $\varphi$ and $\widetilde{\varphi}$, respectively.

Let $\varphi^{\prime}: S \mathcal{A}_{N} \rightarrow \mathcal{D}$ be the $*$-homomorphism defined by

$$
\varphi^{\prime}(f)(s)= \begin{cases}\theta(f(s)) & \text { for } s \in[0,1), \\ \theta(f(-s)) & \text { for } s \in(-1,0] .\end{cases}
$$

Since $\pi^{\prime}+\theta: \mathcal{A}_{N} \rightarrow \mathcal{T}_{A, B}$ coincides with the embedding map, we have $\psi \circ \iota=\varphi+\varphi^{\prime}$. The element of $K K\left(S \mathcal{A}_{N}, \mathcal{D}\right)$ induced by the $*$-homomorphism $\varphi^{\prime}$ is 0 because it factors through the contractible $C^{*}$-algebra $C_{0}\left([0,1), \mathcal{A}_{N}\right)$. Hence the element of $K K\left(S \mathcal{A}_{N}, \mathcal{D}\right)$ induced by $\psi \circ \iota$ coincides with the one by $\varphi$. 
Since the representation $\rho \circ \theta$ vanishes on $\ell^{2}\left(\mathcal{F}^{*}\right), \widetilde{\pi}+(\rho \circ \theta)$ is a representation of $\mathcal{T}_{A, B}$ on $\ell^{2}\left(\mathcal{F}^{*, *}\right)$. The two representations $\tilde{\pi}+(\rho \circ \theta)$ and $\rho$ coincide for $\left\{p_{i}, u_{i}\right\}_{i=1}^{N}$, but

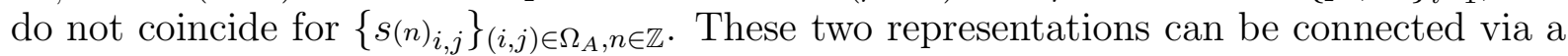
continuous path $\left\{\rho_{t}\right\}_{t \in[0,1]}$ of representations defined by $\rho_{t}\left(p_{i}\right)=\rho\left(p_{i}\right), \rho_{t}\left(u_{i}\right)=\rho\left(u_{i}\right)$ and

$$
\rho_{t}\left(s(n)_{i, j}\right):=\sqrt{1-t^{2}} \widetilde{\pi}\left(s(n)_{i, j}\right)+t \rho\left(s(n)_{i, j} p_{j}^{\prime}\right)+\rho\left(\theta\left(s(n)_{i, j}\right)\right)
$$

for generators $\left\{p_{i}, u_{i}, s(n)_{i, j}\right\}$ of $\mathcal{T}_{A, B}$ and $t \in[0,1]$. It is routine to check that for each $t \in$ $[0,1]$, the $*$-homomorphism $\rho_{t}: \mathcal{T}_{A, B} \rightarrow \mathcal{L}$ is well-defined and satisfies $\rho_{t}(x)-\rho(\theta(x)) \in \mathcal{K}$ for all $x \in \mathcal{T}_{A, B}$. We define a homotopy $\left\{\widetilde{\varphi}_{t}\right\}_{t \in[0,1]}$ of $*$-homomorphisms from $S \mathcal{T}_{A, B}$ to $\widetilde{\mathcal{D}}$ by

$$
\widetilde{\varphi}_{t}(f)(s)= \begin{cases}\rho_{t}(f(s)) & \text { for } s \in[0,1) \\ \rho(\theta(f(-s))) & \text { for } s \in(-1,0]\end{cases}
$$

Similarly as above, we can see that the two elements $\widetilde{\varphi}$ and $\widetilde{\varphi}_{0}$ induce the same element in $K K\left(S \mathcal{T}_{A, B}, \widetilde{\mathcal{D}}\right)$ because $\widetilde{\varphi}_{0}$ is the sum of $\widetilde{\varphi}$ and a $*$-homomorphism factoring through a contractible $C^{*}$-algebra. Since $\widetilde{\varphi}_{0}$ and $\widetilde{\varphi}_{1}=\rho \circ \psi$ also induce the same element, the element in $K K\left(S \mathcal{T}_{A, B}, \widetilde{\mathcal{D}}\right)$ induced by $\widetilde{\varphi}$ coincides with the one induced by $\rho \circ \psi$. This completes the proof of Lemma 7.6.

\section{Appendix B. $\Gamma_{A, B}$-EQUivariant SPlitTing MAPS}

In this appendix, we prove that the $\Gamma_{A, B}$-equivariant exact sequences

$$
\begin{aligned}
& 0 \longrightarrow \operatorname{coker}(I-A) \longrightarrow K_{0}\left(\mathcal{O}_{A, B}\right) \longrightarrow \operatorname{ker}(I-B) \longrightarrow 0 \\
& 0 \longrightarrow \operatorname{coker}(I-B) \longrightarrow K_{1}\left(\mathcal{O}_{A, B}\right) \longrightarrow \operatorname{ker}(I-A) \longrightarrow 0
\end{aligned}
$$

in Proposition 7.10 have $\Gamma_{A, B}$-equivariant splitting maps.

We first see what the homomorphism $K_{0}\left(\mathcal{O}_{A, B}\right) \rightarrow \operatorname{ker}(I-B)$ is. An element in $K_{0}\left(\mathcal{O}_{A, B}\right)$ can be presented by an unitary $u \in C\left([0,1], M_{n}\left(\widetilde{\mathcal{O}}_{A, B}\right)\right)$ with $u(0)=u(1)=1$ for some positive integer $n$, where $\widetilde{\mathcal{O}}_{A, B}$ is the minimal unital $C^{*}$-algebra containing $\mathcal{O}_{A, B}$. We can lift $u \in C\left([0,1], M_{n}\left(\widetilde{\mathcal{O}}_{A, B}\right)\right)$ to an unitary $\bar{u} \in C\left([0,1], M_{n}\left(\widetilde{\mathcal{T}}_{A, B}\right)\right)$ with $\bar{u}(0)=1$. Then $\bar{u}(1)$ is a unitary in $M_{n}\left(\widetilde{\mathcal{J}}_{A, B}\right)$ which defines an element in $K_{1}\left(\mathcal{J}_{A, B}\right)$. In this way, we have the homomorphism $K_{0}\left(\mathcal{O}_{A, B}\right) \rightarrow K_{1}\left(\mathcal{J}_{A, B}\right) \cong \mathbb{Z}^{N}$, and by the proof of Proposition 7.10 we can see that the image of this homomorphism is $\operatorname{ker}(I-B) \subset \mathbb{Z}^{N}$.

For $(i, j) \in \Omega_{A}$, we set $u_{i, j}=\sum_{n=1}^{A_{i, j}} s(n+1)_{i, j} s(n)_{i, j}^{*} \in \mathcal{T}_{A, B}$. Note that $\left\{u_{i}^{\prime}\right\}_{i=1}^{N} \cup\left\{u_{i, j}\right\}_{(i, j) \in \Omega_{A}}$ is a family of mutually orthogonal partial unitaries in $\mathcal{T}_{A, B}$. Hence $\left\{\widetilde{u}_{i}^{\prime}\right\}_{i=1}^{N} \cup\left\{\widetilde{u}_{i, j}\right\}_{(i, j) \in \Omega_{A}}$ is a family of mutually commutative unitaries in $\widetilde{\mathcal{T}}_{A, B}$. We can see that these unitaries commute with mutually commutative unitaries $\left\{\widetilde{u}_{i}\right\}_{i=1}^{N}$ from the equations $\widetilde{u}_{i}=$ $\widetilde{u}_{i}^{\prime} \times \prod_{j \in \Omega_{A}(i)} \widetilde{u}_{i, j}^{B_{i, j}}$.

Lemma B.1. For $\sum_{i=1}^{N} n_{i} e_{i} \in \operatorname{ker}(I-B)$, we have $\prod_{(i, j) \in \Omega_{A}}\left(\widetilde{u}_{j} \widetilde{u}_{i, j}^{-1}\right)^{n_{i} B_{i, j}}=\prod_{i=1}^{N}\left(\widetilde{u}_{i}^{\prime}\right)^{n_{i}}$. 
Proof. Take $\sum_{i=1}^{N} n_{i} e_{i} \in \operatorname{ker}(I-B)$. Then for each $j \in\{1,2, \ldots, N\}$, we have $n_{j}=$ $\sum_{i=1}^{N} n_{i} B_{i, j}$. Hence we obtain

$$
\begin{aligned}
\prod_{(i, j) \in \Omega_{A}}\left(\widetilde{u}_{j} \widetilde{u}_{i, j}^{-1}\right)^{n_{i} B_{i, j}} & =\prod_{i, j=1}^{N} \widetilde{u}_{j}^{n_{i} B_{i, j}} \times \prod_{i=1}^{N}\left(\prod_{j \in \Omega_{A}(i)} \widetilde{u}_{i, j}^{B_{i, j}}\right)^{-n_{i}} \\
& =\prod_{j=1}^{N} \widetilde{u}_{j}^{\sum_{i=1}^{N} n_{i} B_{i, j}} \times \prod_{i=1}^{N}\left(\widetilde{u}_{i}\left(\widetilde{u}_{i}^{\prime}\right)^{-1}\right)^{-n_{i}} \\
& =\prod_{j=1}^{N} \widetilde{u}_{j}^{n_{j}} \times \prod_{i=1}^{N}\left(\widetilde{u}_{i}^{-n_{i}}\left(\widetilde{u}_{i}^{\prime}\right)^{n_{i}}\right) \\
& =\prod_{i=1}^{N}\left(\widetilde{u}_{i}^{\prime}\right)^{n_{i}} .
\end{aligned}
$$

The action $\Gamma_{A, B} \curvearrowright \mathcal{T}_{A, B}$ naturally induces an action $\Gamma_{A, B} \curvearrowright C\left([0,1], M_{2}\left(\widetilde{\mathcal{T}}_{A, B}\right)\right)$.

Lemma B.2. There exists a family of unitaries $\left\{U_{i, j}\right\}_{(i, j) \in \Omega_{A}}$ in $C\left([0,1], M_{2}\left(\widetilde{\mathcal{T}}_{A, B}\right)\right)$ such that

$$
U_{i, j}(0)=\left(\begin{array}{ll}
1 & 0 \\
0 & 1
\end{array}\right), \quad U_{i, j}(1)=\left(\begin{array}{cc}
\widetilde{u}_{j} \widetilde{u}_{i, j}^{-1} & 0 \\
0 & 1
\end{array}\right)
$$

and $\gamma\left(U_{i, j}\right)=U_{\gamma(i), \gamma(j)}$ for $(i, j) \in \Omega_{A}$ and $\gamma \in \Gamma_{A, B}$.

Proof. Take $(i, j) \in \Omega_{A}$. We define a projection $q_{i, j} \in \mathcal{T}_{A, B}$ and a partial unitary $v_{i, j} \in \mathcal{T}_{A, B}$ with $v_{i, j}^{0}=q_{i, j}$ by

$$
q_{i, j}:=\sum_{n=1}^{A_{i, j}} s(n)_{i, j} s(n)_{i, j}^{*}, \quad v_{i, j}:=s\left(A_{i, j}\right)_{i, j} s(1)_{i, j}^{*}+\sum_{n=2}^{A_{i, j}} s(n-1)_{i, j} s(n)_{i, j}^{*} .
$$

We have $v_{i, j}^{A_{i, j}}=q_{i, j}$. We define $w_{i, j} \in C\left([0,1], \mathcal{T}_{A, B}\right)$ by

$$
w_{i, j}(t):=u_{i, j} \times\left(\frac{1}{A_{i, j}} \sum_{m=0}^{A_{i, j}-1}\left(\sum_{n=1}^{A_{i, j}} e^{2 \pi \sqrt{-1} \frac{n}{A_{i, j}}(t-m)}\right) v_{i, j}^{m}\right)
$$

for $t \in[0,1]$. Then for each $t \in[0,1], w_{i, j}(t) \in \mathcal{T}_{A, B}$ is a partial unitary with $w_{i, j}(t)^{0}=q_{i, j}$. We have $w_{i, j}(0)=u_{i, j}$ and

$$
w_{i, j}(1)=u_{i, j} v_{i, j}=s(1)_{i, j} u_{j} s(1)_{i, j}^{*}+\sum_{n=2}^{A_{i, j}} s(n)_{i, j} s(n)_{i, j}^{*} .
$$

Let us define a projection $Q_{i, j} \in M_{2}\left(\mathcal{T}_{A, B}\right)$ by

$$
Q_{i, j}:=\left(\begin{array}{cc}
q_{i, j} & 0 \\
0 & p_{j}
\end{array}\right) .
$$

We define $V_{i, j} \in C\left([0,1], M_{2}\left(\mathcal{T}_{A, B}\right)\right)$ by

$$
V_{i, j}(t):=\left(\begin{array}{cc}
\sqrt{1-t^{2}} s(1)_{i, j}\left(s(1)_{i, j}\right)^{*}+\sum_{n=2}^{A_{i, j}} s(n)_{i, j} s(n)_{i, j}^{*} & -t s(1)_{i, j} \\
t\left(s(1)_{i, j}\right)^{*} & \sqrt{1-t^{2}} p_{j}
\end{array}\right)
$$


for $t \in[0,1]$. Then for each $t \in[0,1], V_{i, j}(t) \in M_{2}\left(\mathcal{T}_{A, B}\right)$ is a partial unitary with $V_{i, j}(t)^{0}=$ $Q_{i, j}$. We also have $V_{i, j}(0)=Q_{i, j}$. Now we define a unitary $U_{i, j} \in C\left([0,1], M_{2}\left(\widetilde{\mathcal{T}}_{A, B}\right)\right)$ by

$$
\begin{array}{r}
U_{i, j}(t):=\left(\begin{array}{cc}
\sqrt{1-t^{2}} & -t \\
t & \sqrt{1-t^{2}}
\end{array}\right)\left(V_{i, j}(t)\left(\begin{array}{cc}
w_{i, j}(t) & 0 \\
0 & p_{j}
\end{array}\right) V_{i, j}(t)^{*}+\left(\begin{array}{cc}
1 & 0 \\
0 & 1
\end{array}\right)-Q_{i, j}\right) \\
\left(\begin{array}{cc}
\sqrt{1-t^{2}} & t \\
-t & \sqrt{1-t^{2}}
\end{array}\right)\left(\begin{array}{cc}
\widetilde{u}_{i, j}^{-1} & 0 \\
0 & 1
\end{array}\right) .
\end{array}
$$

Then the family of unitaries $\left\{U_{i, j}\right\}_{(i, j) \in \Omega_{A}}$ satisfies the desired conditions.

The same formulae as the ones in $\mathcal{T}_{A, B}$ define $u_{i, j} \in \mathcal{O}_{A, B}$ and $U_{i, j} \in C\left([0,1], M_{2}\left(\widetilde{\mathcal{O}}_{A, B}\right)\right)$ which are nothing but the images under the surjection $\mathcal{T}_{A, B} \rightarrow \mathcal{O}_{A, B}$. Note that although $\left\{U_{i, j}(1)\right\}_{(i, j) \in \Omega_{A}}$ commute with each others, $\left\{U_{i, j}\right\}_{(i, j) \in \Omega_{A}}$ need not. However we can show that these unitaries commute with each others "up to homotopy" without changing the values at the two ends.

Lemma B.3. Let $\mathcal{A}$ be a unital $C^{*}$-algebra, and $u, v$ be unitaries in $\mathcal{A}$. Suppose that $u, v$ can be written as $u=\prod_{i=1}^{K} \widetilde{w}_{i}^{k_{i}}, v=\prod_{i=1}^{K} \widetilde{w}_{i}^{l_{i}}$ for $k_{i}, l_{i} \in \mathbb{Z}$ and a family $\left\{w_{i}\right\}_{i=1}^{K}$ of mutually orthogonal partial unitaries. Then there exists a homotopy $[-1,1] \ni s \mapsto a_{s} \in \mathcal{A}$ with $a_{-1}=1-v, a_{1}=u(1-v)$ such that $u^{*} a_{s}+v a_{-s}^{*}=0$ and $a_{s} a_{s}^{*}=a_{s}^{*} a_{s}=2-v-v^{*}$ hold for all $s \in[-1,1]$.

Proof. We may assume that $u=w^{k}$ and $v=w^{l}$ for a unitary $w \in \mathcal{A}$ and $k, l \in \mathbb{Z}$. We set $a_{s}=f_{s}(w)$ for $s \in[-1,1]$ where $f_{s} \in C(\mathbb{T})$ is defined by

$$
f_{s}\left(e^{2 \pi \sqrt{-1} t}\right):=e^{(s+1) \pi \sqrt{-1} k t}\left(1-e^{2 \pi \sqrt{-1} l t}\right)
$$

for $t \in[0,1]$. Now it is routine to check that the continuous path $[-1,1] \ni s \mapsto a_{s} \in \mathcal{A}$ satisfies the desired conditions.

Lemma B.4. Let $\mathcal{A}$ be a unital $C^{*}$-algebra. Let $u, v \in C([0,1], \mathcal{A})$ be two continuous paths of unitaries such that $u(0)=v(0)=1$ and $u(1), v(1) \in \mathcal{A}$ satisfy the assumption in Lemma B.3. Then there exists a homotopy $[-1,1] \ni s \mapsto W_{s} \in C\left([0,1], M_{2}(\mathcal{A})\right)$ of unitaries such that

$$
W_{-1}(t)=\left(\begin{array}{cc}
u(t) v(t) & 0 \\
0 & 1
\end{array}\right), \quad W_{1}(t)=\left(\begin{array}{cc}
v(t) u(t) & 0 \\
0 & 1
\end{array}\right)
$$

for all $t \in[0,1]$ and

$$
W_{s}(0)=\left(\begin{array}{ll}
1 & 0 \\
0 & 1
\end{array}\right), \quad W_{s}(1)=\left(\begin{array}{cc}
u(1) v(1) & 0 \\
0 & 1
\end{array}\right)=\left(\begin{array}{cc}
v(1) u(1) & 0 \\
0 & 1
\end{array}\right)
$$

for all $s \in[-1,1]$.

Proof. For $s \in[0,1]$, we define unitaries $W_{-s}^{\prime}, W_{s}^{\prime} \in C\left([0,1], M_{2}(\mathcal{A})\right)$ by

$$
\begin{aligned}
W_{-s}^{\prime}(t) & =\left(\begin{array}{cc}
u(t) & 0 \\
0 & 1
\end{array}\right)\left(\begin{array}{cc}
s & -\sqrt{1-s^{2}} \\
\sqrt{1-s^{2}} & s
\end{array}\right)\left(\begin{array}{cc}
v(t) & 0 \\
0 & 1
\end{array}\right)\left(\begin{array}{cc}
s & \sqrt{1-s^{2}} \\
-\sqrt{1-s^{2}} & s
\end{array}\right), \\
W_{s}^{\prime}(t) & =\left(\begin{array}{cc}
s & -\sqrt{1-s^{2}} \\
\sqrt{1-s^{2}} & s
\end{array}\right)\left(\begin{array}{cc}
v(t) & 0 \\
0 & 1
\end{array}\right)\left(\begin{array}{cc}
s & \sqrt{1-s^{2}} \\
-\sqrt{1-s^{2}} & s
\end{array}\right)\left(\begin{array}{cc}
u(t) & 0 \\
0 & 1
\end{array}\right) .
\end{aligned}
$$

Then the two definitions of $W_{0}^{\prime}$ coincide, and we get a homotopy $[-1,1] \ni s \mapsto W_{s}^{\prime} \in$ $C\left([0,1], M_{2}(\mathcal{A})\right)$ of unitaries. This homotopy satisfies the desired conditions except the 
last condition on $t=1$. Since $u(1), v(1) \in \mathcal{A}$ satisfy the assumption in Lemma B.3, we can find a homotopy $[-1,1] \ni s \mapsto a_{s} \in \mathcal{A}$ with $a_{-1}=1-v(1), a_{1}=u(1)(1-v(1))$ such that $u(1)^{*} a_{s}+v(1) a_{-s}^{*}=0$ and $a_{s} a_{s}^{*}=a_{s}^{*} a_{s}=2-v(1)-v(1)^{*}$ hold for all $s \in[-1,1]$. We define a homotopy $[-1,1] \ni s \mapsto W_{s}^{\prime \prime} \in C\left([0,1], M_{2}(\mathcal{A})\right)$ of unitaries by

$$
W_{s}^{\prime \prime}(t)=\left(\begin{array}{cc}
u(1)\left(t^{2} v(1)+\left(1-t^{2}\right)\right) & t \sqrt{1-t^{2}} a_{-s} \\
t \sqrt{1-t^{2}} a_{s} & t^{2}+\left(1-t^{2}\right) v(1)
\end{array}\right)
$$

for $s \in[-1,1]$ and $t \in[0,1]$. Then this homotopy satisfies

$$
W_{s}^{\prime \prime}(0)=\left(\begin{array}{cc}
u(1) & 0 \\
0 & v(1)
\end{array}\right)=W_{0}^{\prime}(1), \quad W_{s}^{\prime \prime}(1)=\left(\begin{array}{cc}
u(1) v(1) & 0 \\
0 & 1
\end{array}\right)
$$

for all $s \in[-1,1]$, and $W_{-1}^{\prime \prime}(t)=W_{-t}^{\prime}(1)$ and $W_{1}^{\prime \prime}(t)=W_{t}^{\prime}(1)$ for $t \in[0,1]$. We define a homotopy $[-1,1] \ni s \mapsto W_{s} \in C\left([0,1], M_{2}(\mathcal{A})\right)$ of unitaries by

$$
W_{s}(t)= \begin{cases}W_{s}^{\prime}((2 /(1+|s|)) t) & \text { for } 0 \leq t \leq(1+|s|) / 2 \\ W_{s /(2 t-1)}^{\prime \prime}(2 t-1) & \text { for }(1+|s|) / 2<t \leq 1\end{cases}
$$

This homotopy satisfies the desired conditions.

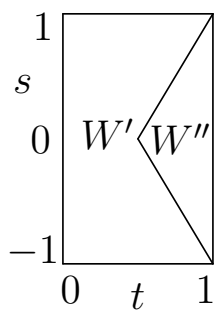

Remark B.5. In the lemma above, one can weaken the assumptions on $u(1), v(1) \in \mathcal{A}$. However, the assumption that $u(1) v(1)=v(1) u(1)$ is too weak to get the conclusion, and we can find a counterexample in $\mathcal{A}=M_{2}\left(C\left(\mathbb{T}^{2}\right)\right)$ for example. Note that the condition that two unitaries $u$ and $v$ commute with each others is necessary, but not sufficient for the conclusion of Lemma B.3.

Now we construct a $\Gamma_{A, B}$-equivariant splitting map $\operatorname{ker}(I-B) \rightarrow K_{0}\left(\mathcal{O}_{A, B}\right)$ for the surjection $K_{0}\left(\mathcal{O}_{A, B}\right) \rightarrow \operatorname{ker}(I-B)$ in Proposition 7.10. For $f=\sum_{i=1}^{N} n_{i} e_{i} \in \operatorname{ker}(I-B)$, we define a unitary

$$
U_{f}:=\prod_{(i, j) \in \Omega_{A}} U_{i, j}^{n_{i} B_{i, j}} \in C\left([0,1], M_{2}\left(\widetilde{\mathcal{O}}_{A, B}\right)\right) .
$$

Then by the computation in Lemma B.1 we have $U_{f}(0)=U_{f}(1)=1$. Thus this unitary defines an element $\left[U_{f}\right] \in K_{0}\left(\mathcal{O}_{A, B}\right)$. Although the definition of a unitary $U_{f} \in C\left([0,1], M_{2}\left(\widetilde{\mathcal{O}}_{A, B}\right)\right)$ depends on the order of the product above, Lemma B.4 shows that the element $\left[U_{f}\right] \in K_{0}\left(\mathcal{O}_{A, B}\right)$ does not depend because $U_{i, j}(0)=1$ for all $(i, j) \in \Omega_{A}$ and $\left\{U_{i, j}(1)\right\}_{(i, j) \in \Omega_{A}}$ satisfies the assumption in Lemma B.3. From this fact, we see that the map

$$
\operatorname{ker}(I-B) \ni f \mapsto\left[U_{f}\right] \in K_{0}\left(\mathcal{O}_{A, B}\right)
$$

is a well-defined group homomorphism. We can also see that this homomorphism is $\Gamma_{A, B^{-}}$ equivariant by Lemma B.2. We will see that this homomorphism is a splitting map for the surjection $K_{0}\left(\mathcal{O}_{A, B}\right) \rightarrow \operatorname{ker}(I-B)$. The same formula as $U_{f}$ defines a lifting of $U_{f}$ in $C\left([0,1], M_{2}\left(\widetilde{\mathcal{T}}_{A, B}\right)\right)$ whose value at 1 is $\prod_{i=1}^{N}\left(\widetilde{u}_{i}^{\prime}\right)^{n_{i}}$ by Lemma B.1. We see that $\left[\prod_{i=1}^{N}\left(\widetilde{u}_{i}^{\prime}\right)^{n_{i}}\right] \in K_{1}\left(\mathcal{J}_{A, B}\right)$ corresponds to $f=\sum_{i=1}^{N} n_{i} e_{i} \in \operatorname{ker}(I-B) \subset \mathbb{Z}^{n}$ via the natural isomorphism $K_{1}\left(\mathcal{J}_{A, B}\right) \cong \mathbb{Z}^{n}$. Hence the map $f \mapsto\left[U_{f}\right]$ is a splitting map for the surjection $K_{0}\left(\mathcal{O}_{A, B}\right) \rightarrow \operatorname{ker}(I-B)$. 
In a similar way as above, one can show that the other surjection $K_{1}\left(\mathcal{O}_{A, B}\right) \rightarrow \operatorname{ker}(I-A)$ in Proposition 7.10 also has a $\Gamma_{A, B}$-equivariant splitting map. However for this surjection, we can use the well-known computations of $K$-groups of Cuntz-Krieger algebras $\mathcal{O}_{A}$ as follows (cf. [EL2]).

Proposition B.6. Let $A, B \in M_{N}(\mathbb{Z})$ satisfy the condition (0). Then $K_{0}\left(\mathcal{O}_{A}\right)$ and $K_{1}\left(\mathcal{O}_{A}\right)$ are isomorphic to $\operatorname{coker}(I-A)$ and $\operatorname{ker}(I-A)$ as $\Gamma_{A, B}$-modules respectively, and the inclusion $\mathcal{O}_{A} \hookrightarrow \mathcal{O}_{A, B}$ induces the injection coker $(I-A) \rightarrow K_{0}\left(\mathcal{O}_{A, B}\right)$ in Proposition 7.10 and a $\Gamma_{A, B}$-equivariant map $\operatorname{ker}(I-A) \rightarrow K_{1}\left(\mathcal{O}_{A, B}\right)$ which is a splitting map for the surjection $K_{1}\left(\mathcal{O}_{A, B}\right) \rightarrow \operatorname{ker}(I-A)$ in Proposition \%.10.

Proof. The proof is very similar to the one of Proposition 7.10.

Let $\mathcal{T}_{A} \subset \mathcal{T}_{A, B}$ be the $C^{*}$-subalgebra generated by $\left\{p_{i}\right\}_{i=1}^{N}$ and $\left\{s(n)_{i, j}\right\}_{(i, j) \in \Omega_{A}, n \in\left\{1,2, \ldots, A_{i, j}\right\}}$. We set $\mathcal{J}_{A}:=\mathcal{T}_{A} \cap \mathcal{J}_{A, B}$. Then we get the following $\Gamma_{A, B}$-equivariant commutative diagram with exact rows

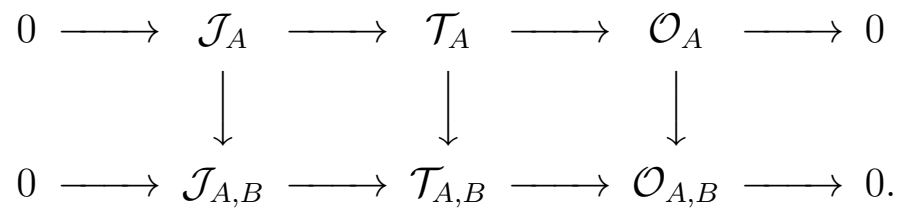

Let $\mathcal{B}_{N} \subset \mathcal{A}_{N}$ be the $C^{*}$-subalgebra generated by $\left\{p_{i}\right\}_{i=1}^{N}$. Thus $\mathcal{B}_{N} \cong \mathbb{C}^{N}$. In a similar way as Lemma 7.6 and Lemma 7.7 , we can show that the restrictions $\mathcal{B}_{N} \rightarrow \mathcal{T}_{A}$ and $\mathcal{B}_{N} \rightarrow \mathcal{J}_{A}$ of $*$-homomorphisms in the two lemmas are $K K$-equivalences. From these facts and an easy diagram chasing, we get the conclusions.

This finishes the proofs of the existence of the $\Gamma_{A, B^{-}}$equivariant splitting maps for the two surjections in Proposition 7.10, and hence finishes the proof of Proposition 7.10.

\section{REFERENCES}

[BKP] Benson, D. J.; Kumjian, A.; Phillips N. C. Symmetries of Kirchberg algebras. Canad. Math. Bull. 46 (2003), no. 4, 509-528.

[Bl] Blackadar, B. K-theory for operator algebras. Second edition. Mathematical Sciences Research Institute Publications, 5. Cambridge University Press, Cambridge, 1998.

[Br] Brown, K. S. Cohomology of groups. Graduate Texts in Mathematics, 87. Springer-Verlag, New York, 1994.

[CK] Cuntz, J.; Krieger, W. A class of $C^{*}$-algebras and topological Markov chains. Invent. Math. 56 (1980), no. 3, 251-268.

[D] Deaconu, V. Generalized Cuntz-Krieger algebras. Proc. Amer. Math. Soc. 124 (1996), no. 11, 3427-3435.

[DLRZ] Doplicher, S.; Longo, R.; Roberts, J. E.; Zsido, L. A remark on quantum group actions and nuclearity. Rev. Math. Phys. 14 (2002), no. 7-8, 787-796.

[EL1] Exel, R.; Laca, M. Cuntz-Krieger algebras for infinite matrices. J. Reine Angew. Math. 512 (1999), 119-172.

[EL2] Exel, R.; Laca, M. The K-theory of Cuntz-Krieger algebras for infinite matrices. K-Theory 19 (2000), no. 3, 251-268.

[I] Izumi, M. Finite group actions on $C^{*}$-algebras with the Rohlin property. II. Adv. Math. 184 (2004), no. 1, 119-160.

[Ka1] Katsura, T. A class of $C^{*}$-algebras generalizing both graph algebras and homeomorphism $C^{*}$ algebras I, fundamental results. Trans. Amer. Math. Soc. 356 (2004), no. 11, 4287-4322.

[Ka2] Katsura, T. A construction of $C^{*}$-algebras from $C^{*}$-correspondences. Advances in Quantum Dynamics, 173-182, Contemp. Math., 335, Amer. Math. Soc., Providence, RI, 2003. 
[Ka3] Katsura, T. On $C^{*}$-algebras associated with $C^{*}$-correspondences. J. Funct. Anal. 217 (2004), no. $2,366-401$.

[Ka4] Katsura, T. A class of $C^{*}$-algebras generalizing both graph algebras and homeomorphism $C^{*}$ algebras IV, pure infiniteness. Preprint 2005, math.OA/0509343.

[Ka5] Katsura, T. Permutation presentations of modules over finite groups. Preprint 2006, math.GR/0608091.

[Ki] Kirchberg, E. The classification of purely infinite $C^{*}$-algebras using Kasparov's theory. Preprint 1994.

[KPR] Kumjian, A.; Pask, D.; Raeburn, I. Cuntz-Krieger algebras of directed graphs. Pacific J. Math. 184 (1998), no. 1, 161-174.

[Ph] Phillips, N. C. A classification theorem for nuclear purely infinite simple $C^{*}$-algebras. Doc. Math. 5 (2000), 49-114.

[Pi] Pimsner, M. V. A class of $C^{*}$-algebras generalizing both Cuntz-Krieger algebras and crossed products by Z. Free probability theory, 189-212, Fields Inst. Commun., 12, Amer. Math. Soc., Providence, RI, 1997.

[Ra] Raeburn, I. Graph algebras. CBMS Regional Conference Series in Mathematics, 103. Published for the Conference Board of the Mathematical Sciences, Washington, DC; by the American Mathematical Society, Providence, RI, 2005.

[Rø] Rørdam, M. Classification of nuclear $C^{*}$-algebras. Encyclopaedia of Mathematical Sciences, 126. Operator Algebras and Non-commutative Geometry, 7. Springer-Verlag, Berlin, 2002, pages 1145.

[Sp] Spielberg, J. Non-cyclotomic Presentations of Modules and Prime-order Automorphisms of Kirchberg Algebras. Preprint 2005, math.OA/0504287.

[Z] Zhang, S. Certain $C^{*}$-algebras with real rank zero and their corona and multiplier algebras. I. Pacific J. Math. 155 (1992), no. 1, 169-197.

Department of Mathematics, Hokkaido University, Kita 10, Nishi 8, Kita-Ku, Sapporo, 060-0810, JAPAN

E-mail address: katsura@math.sci.hokudai.ac.jp 